\title{
Analysis of membrane locking in hp FEM for a cylindrical shell
}

\section{Report}

Author(s):

Gerdes, K.; Matache, Ana-Maria; Schwab, Christoph

Publication date:

1997-07

Permanent link:

https://doi.org/10.3929/ethz-a-004284910

Rights / license:

In Copyright - Non-Commercial Use Permitted

Originally published in:

SAM Research Report 1997-09 


\title{
Analysis of membrane locking \\ in \\ $h p$ FEM for a cylindrical shell
}

K. Gerdes, A. M. Matache and C. Schwab

\author{
Seminar for Applied Mathematics \\ ETH Zürich \\ Rämistr. 101 \\ CH-8092 Zürich, Switzerland
}

\begin{abstract}
In this paper we analyze the performance of the $h p$-Finite Element Method for a cylindrical shell problem. Our theoretical investigations show that the $h p$ approximation converges exponentially, provided that boundary layers stemming from the edge effect are resolved. The numerical results illustrate the mesh independence of the exponential convergence of the $h p-\mathrm{FEM}$.
\end{abstract}

Mathematics Subject Classification (1991): Primary: 65N30, 73K15; Secondary: 65M70, $65 \mathrm{~L} 70$. 


\section{Introduction}

The largest class of problems that are numerically solved in industrial FE analyses in the U.S. are linear, static problems for solids, and among these, again a major part is taken by thin structures, such as beams, plates and shells [3]. Owing to the singular perturbation character of these problems, it was found early [8] that conventional FEM do not converge satisfactorily due to locking effects. A large body of literature has been devoted to this phenomenon and to possible remedies. In the case of plate bending, there are by now methodologies (such as the "MITC" reduced shear elements of Bathe and Dvorkin [8]) which allow to resolve plate bending problems satisfactorily, even at small values of the plate thickness $t$.

In the case of shells, the locking phenomena are much more complex and to date there is no mathematically validated approach for the complete removal of shear and/ or membrane locking that works equally well in bending or membrane dominated cases. Worse, there is evidence that this cannot be overcome [6].

In special situations, of course, there are ways to alleviate/ reduce membrane locking by methodologies similar to those that are successful in the plate bending case [2], [10] and which are based on modifications of the variational principle (mixed methods; "reduced integration", etc). These remarks refer to the $h$-version FEM where convergence is achieved by letting the meshwidth $h$ tend to zero to achieve convergence.

In the last decade, the $p$ - and $h p$-versions of the FEM have emerged [1]. Here convergence is achieved by increasing the polynomial degree rather than by mesh-refinement. It has been shown in a series of papers by J. Pitkäranta and co-workers ([10], [11] and the references there) that $h$-FEM based on the standard displacement variational principle and high polynomial degree appear to be either free of or substantially less susceptible to membrane locking, even without special changes of the variational principle.

At present, the development of a commercial $h p$-shell implementation for general surfaces and also for laminated shells is under way in the code STRESSCHECK at ESRD Res. Corp. ${ }^{1}$ and several other major FE-software vendors are including $p$ and $h p$-capabilities into their codes.

The purpose of the present paper is to analyse the performance of the $h p$-FEM for a class of benchmark problems for a shell model of Naghdi -type, i.e. including shear deformation ${ }^{2}$ computationally as well as theoretically. Our main theoretical result shows that the $h p$-FEM

\footnotetext{
${ }^{1} 7750$ Clayton Road, Suite 204 St. Louis, Missouri 63117 ,USA

${ }^{2}$ We do not address here the dimension-reduction error; for these issues, we refer to [4] and the references there.
} 
based on the principle of virtual work in the displacements, increasing polynomial degree and on boundary layer resolution via anisotropic meshes leads to exponential rates of convergence, i.e. the relative FE-error in the shell energy norm ||$|\cdot|||$ satisfies

$$
\frac{\left\|\underline{\underline{U}}-\underline{\tilde{U}}^{h p}|\||\right.}{\|\underline{U} \mid\|} \leq C t^{\beta} e^{-\eta p},
$$

where $C, \beta$ are independent of $h, p$ and the thickness $t$, and where $t^{\beta},-1 \leq \beta \leq 0$ is a locking factor. The estimate (1.1) is not robust, i.e. the convergence rate depends on $t$, but due to the exponential term $e^{-\eta p}$ we expect that for increasing $p$ the $t$-sensitivity is practically negligible; this is indeed the case in the practical range of $t$ and $p$, as we show in numerical experiments: relative energy errors below $1 \%$ are easily achievable, even for $t$ as small as $10^{-3}$. We demonstrate this in detail for the cylindrical shell benchmark problems of [11]; although here the geometry is relatively simple, these problems exhibit a wide range of behaviours, from membrane to bending dominated. The explicitly available solution of the benchmark problems allows a complete analysis of the $h p$-FEM, resulting in (1.1). We also show in numerical experiments the relative insensitivity of $h p$-FEM with regard to the mesh design: comparable numerical results are obtained for both, triangular and quadrilateral elements, for aligned or general meshes.

This leads us to the conjecture that the exponential rate (1.1) can be achieved for general shells as well; in fact, our computer-implementation can handle such cases easily; the proof of (1.1) in complete generality requires new results on the asymptotics of shells that are not yet available, however.

The outline of the paper is as follows: Section 2 presents the shell problem, the shell model and the $h p$-FEM. The regularity and the asymptotics of the exact solution of the shell model are discussed in detail. Section 3 is devoted to the proof of the error estimate (1.1). Section 4 gives a description and numerical results of our $h p$-FEM for general shells, while Section 5 contains conclusions and perspectives for further research.

\section{The $h p$-FEM and the problem of membrane locking}

In this section we will introduce the 3-D shell model and explain how the dimensionally reduced 2-D model is deduced. We then address the regularity of the solution of the 2-D shell model in detail and present the $h p$-FE discretization. 


\subsection{The three-dimensional shell model}

In the cylindrical coordinate system $(x, \alpha, z)$ the shell occupies the region:

$$
\Omega_{t}=\left\{(x, \alpha, z) \in \mathbb{R}^{3}:(x, \alpha) \in \omega,|z|<\frac{t}{2}\right\},
$$

where $\omega=(-1,1) \times(0,2 \pi]$ is the parametrization domain of the midsurface. The shell is assumed to be loaded on its outer surface by a normal pressure distribution $f=f_{0} \cos k \alpha$, which is constant with respect to the axial variable $x$, such that the oscillating factor $k$ is a bounded integer (for all numerical computations $k=2$ ).

The strain tensor corresponding to a displacement field $\underline{V}^{3 D}$ is defined as $\underline{e}\left(\underline{V}^{3 D}\right)=\left\{e_{i j}\right\}_{i, j=1}^{3}$, which in the 3-D model of linearized, homogeneous and isotropic elasticity takes the form:

$$
\begin{aligned}
e_{11} & =\partial_{x} V_{1}, & e_{22} & =\chi\left(\partial_{\alpha} V_{2}+V_{3}\right), \\
e_{12} & =\frac{1}{2}\left(\chi \partial_{\alpha} V_{1}+\partial_{x} V_{2}\right), & e_{23} & =\frac{1}{2}\left(\partial_{z} V_{2}+\chi\left(\partial_{\alpha} V_{3}-V_{2}\right)\right), \\
e_{13} & =\frac{1}{2}\left(\partial_{z} V_{1}+\partial_{x} V_{3}\right), & e_{33} & =\partial_{z} V_{3} .
\end{aligned}
$$

In the above notation the components $V_{i}$ refer to the local orthonormal basis that corresponds to the curvilinear coordinate system, and $\chi=1 /(1+z)$. We introduce the bilinear form

$$
\mathcal{A}^{3 D}\left(\underline{U}^{3 D}, \underline{V}^{3 D}\right)=D^{-1} \int_{\Omega_{t}}\left\{\lambda \operatorname{tr} \underline{e}\left(\underline{U}^{3 D}\right) \operatorname{tr} \underline{e}\left(\underline{V}^{3 D}\right)+\mu \sum_{i, j=1}^{3} e_{i j}\left(\underline{U}^{3 D}\right) e_{i j}\left(\underline{V}^{3 D}\right)\right\} \chi^{-1} d x d \alpha d z
$$

where $\lambda$ and $\mu$ are Lamé-constants depending on the Young modulus $E>0$ and the Poisson ratio $\nu$, and $\mathrm{D}$ is a scaling factor:

$$
\lambda=\frac{E \nu}{(1+\nu)(1-2 \nu)}, \quad \mu=\frac{E}{1+\nu}, \quad D=\frac{E}{12\left(1-\nu^{2}\right)} .
$$

According to this three-dimensional model, the total energy to be minimized takes the form

$$
\mathcal{F}^{3 D}\left(\underline{U}^{3 D}\right)=\frac{1}{2} \mathcal{A}^{3 D}\left(\underline{U}^{3 D}, \underline{U}^{3 D}\right)-Q^{3 D}\left(\underline{U}^{3 D}\right),
$$

where

$$
Q^{3 D}\left(\underline{U}^{3 D}\right)=D^{-1} \int_{\omega} f(\alpha) \cdot U_{3}\left(x, \alpha, \frac{t}{2}\right) \cdot\left(1+\frac{t}{2}\right) d x d \alpha
$$

The dimensionally reduced shell models to be considered are derived by constraining the components of the displacement field, expressed in terms of the curvilinear coordinates $(x, \alpha, z)$, to be quadratic polynomials with respect to $z$ for each $(x, \alpha) \in \omega$, and by asymptotic expansion and simplification of the resulting energy functional, as shown in [9], Section 3. 


\subsection{The dimensionally reduced shell model}

The shell model is given by the dimensionally reduced shell energy functional

$$
\begin{aligned}
\mathcal{F}_{R}(\underline{U}) & =\frac{t}{2} \int_{\omega}\left[\nu\left(\beta_{11}+\beta_{22}\right)^{2}+(1-\nu) \sum_{i, j=1}^{2} \beta_{i j}^{2}\right] d x d \alpha \\
& +\frac{t^{3}}{24} \int_{\omega}\left[\nu\left(\kappa_{11}+\kappa_{22}\right)^{2}+(1-\nu) \sum_{i, j=1}^{2} \kappa_{i j}^{2}\right] d x d \alpha \\
& +\frac{1-\nu}{4} t \int_{\omega}\left(\rho_{1}^{2}+\rho_{2}^{2}\right) d x d \alpha-\int_{\omega} W f d x d \alpha
\end{aligned}
$$

over the space of admissible displacements

$\mathcal{U}=\left\{\underline{U}=(U, V, W, \Theta, \Phi) \in\left[H^{1}(\omega)\right]^{5}\right.$, satisfying the kinematical constraints at $x= \pm 1$ and the orthogonality condition: $\int_{\omega} \underline{U} \cdot \underline{R} d x d \alpha=0$, for any $\left.\underline{R} \in \underline{\mathcal{R}}\right\}$,

where $\underline{\mathcal{R}}=\left\{\underline{U} \in\left[H^{1}(\omega)\right]^{5}: \mathcal{A}_{R}(\underline{U}, \underline{U})=0\right\}$ is the set of rigid body motions.

Here we denoted by $\beta_{i j}$ the membrane strains, $\kappa_{i j}$ the bending strains, $\rho_{i}$ the transverse shear strains, $1 \leq i, j \leq 2$, and $\omega=(-1,1) \times(0,2 \pi]$ is the parametrization domain of the middle surface of the shell:

$$
\begin{aligned}
& \beta_{11}=\partial_{x} U, \quad \beta_{12}=\frac{1}{2}\left(\partial_{\alpha} U+\partial_{x} V\right), \quad \beta_{22}=\partial_{\alpha} V+W, \\
& \kappa_{11}=\partial_{x} \Theta, \quad \kappa_{12}=\frac{1}{2}\left(\partial_{\alpha} \Theta+\partial_{x} \Phi-\partial_{x} V\right), \quad \kappa_{22}=\partial_{\alpha} \Phi, \\
& \rho_{1}=-\Theta+\partial_{x} W, \quad \rho_{2}=-\Phi-\partial_{\alpha} W-V .
\end{aligned}
$$

We assume for our shell model the following homogeneous boundary conditions:

Case 1: clamped-clamped, $(\underline{U}( \pm 1, \alpha)=0)$,

Case 2: free-free, (no kinematical constraints),

Case 3: simple sliding support at the ends of the cylinder, $(W( \pm 1, \alpha)=0)$.

Let us denote by $\||\cdot \||$ the energy norm of the shell defined as $\| \underline{U} \mid \|=\mathcal{A}_{R}(\underline{U}, \underline{U})^{1 / 2}$, where $\mathcal{A}_{R}(\underline{U}, \underline{U}) / 2$ represents the quadratic part of the energy functional (2.1). Then for every $t>0$ and every load $f$ such that $\int_{\omega} f W d x d \alpha=0 \forall \underline{R} \in \underline{\mathcal{R}}$, there exists a unique minimizer $\underline{U} \in \mathcal{U}$ of $\mathcal{F}_{R}(\underline{U})$.

\subsection{Regularity of the solution of the shell model}

\subsubsection{Separation of variables}

Because of the cylindrical geometry of the domain and of the load we may assume for the displacement field $\underline{U}$ the following tensor-product Ansatz:

$$
\underline{U}(x, \alpha)=\underline{u}(x) \otimes \underline{v}(\alpha),
$$


where

$$
\begin{aligned}
& \underline{u}=\underline{u}(x)=(u, v, w, \theta, \phi)(x), \\
& \underline{v}=\underline{v}(\alpha)=(\cos k \alpha, \sin k \alpha, \cos k \alpha, \cos k \alpha, \sin k \alpha) .
\end{aligned}
$$

The displacement field $\underline{u}(x)=(u, v, w, \theta, \phi)(x)$ minimizes the dimensionally reduced energy functional

$$
\begin{aligned}
\mathcal{F}_{R}(\underline{u}) & =\frac{t}{2} \int_{-1}^{1}\left[\nu\left(\beta_{11}+\beta_{22}\right)^{2}+(1-\nu) \sum_{i, j=1}^{2} \beta_{i j}^{2}\right] d x \\
& +\frac{t^{3}}{24} \int_{-1}^{1}\left[\nu\left(\kappa_{11}+\kappa_{22}\right)^{2}+(1-\nu) \sum_{i, j=1}^{2} \kappa_{i j}^{2}\right] d x \\
& +\frac{1-\nu}{4} t \int_{-1}^{1}\left(\rho_{1}^{2}+\rho_{2}^{2}\right) d x-f_{0} \int_{-1}^{1} w d x,
\end{aligned}
$$

where the bending and membrane strain-tensor $\beta, \kappa$ and the shear vector $\rho$ are defined in terms of the one-dimensional unknown $\underline{u}(x)$ as follows:

$$
\begin{aligned}
& \beta_{11}=u^{\prime}, \quad \beta_{12}=\frac{1}{2}\left(-k u+v^{\prime}\right), \quad \beta_{22}=k v+w, \\
& \kappa_{11}=\theta^{\prime}, \quad \kappa_{12}=\frac{1}{2}\left(-k \theta+\phi^{\prime}-v^{\prime}\right), \quad \kappa_{22}=k \phi, \\
& \rho_{1}=-\theta+w^{\prime}, \quad \rho_{2}=-\phi-k w-v .
\end{aligned}
$$

By $\|\cdot\|_{e}=a(\underline{u}, \underline{u})^{1 / 2}$ we denote the energy norm corresponding to the reduced 1-D model, $a(\underline{u}, \underline{u}) / 2$ being the total deformation energy with respect to the displacement field $\underline{u}$.

The minimizer of the reduced energy functional (2.3) solves the following two point boundary value problem

$$
\begin{aligned}
0 & =u^{\prime \prime}+\nu\left(k v^{\prime}+w^{\prime}\right)+\frac{1-\nu}{2}\left(-k^{2} u+k v^{\prime}\right), \\
0 & =-\nu k u^{\prime}-k^{2} v-k w+\frac{1-\nu}{2}\left(-k u^{\prime}+v^{\prime \prime}\right)-\frac{t^{2}}{24}(1-\nu)\left(-k \theta^{\prime}+\phi^{\prime \prime}-v^{\prime \prime}\right) \\
& +\frac{1-\nu}{2}(-\phi-k w-v), \\
f_{0} & =t\left(\nu u^{\prime}+k v+w\right)-\frac{1-\nu}{2} t\left(-\theta^{\prime}+w^{\prime \prime}-k \phi-k^{2} w-k v\right), \\
0 & =\frac{t^{2}}{12}\left(\theta^{\prime \prime}+\nu k \phi^{\prime}+\frac{1-\nu}{2}\left(-k^{2} \theta+k \phi^{\prime}-k v^{\prime}\right)\right)+\frac{1-\nu}{2}\left(-\theta+w^{\prime}\right), \\
0 & =\frac{t^{2}}{12}\left(-\nu k \theta^{\prime}-k^{2} \phi+\frac{1-\nu}{2}\left(-k \theta^{\prime}+\phi^{\prime \prime}-v^{\prime \prime}\right)\right)-\frac{1-\nu}{2}(\phi+k w+v),
\end{aligned}
$$

and satisfies in each of the three cases the corresponding boundary conditions at $x \in\{-1,1\}$ : Case 1 (clamped edges):

$$
u(x)=v(x)=w(x)=\theta(x)=\phi(x)=0,
$$


Case 2 (free edges):

$$
\begin{aligned}
u^{\prime}(x)+\nu(k v+w)(x) & =0, \\
\left(-k u+v^{\prime}\right)(x)-\frac{t^{2}}{12}\left(-k \theta+\phi^{\prime}-v^{\prime}\right)(x) & =0, \\
-\theta(x)+w^{\prime}(x) & =0, \\
\theta^{\prime}(x)+\nu k \phi(x) & =0, \\
-k \theta(x)+\phi^{\prime}(x)-v^{\prime}(x) & =0,
\end{aligned}
$$

Case 3 (sliding support):

$$
\begin{aligned}
u^{\prime}(x)+\nu(k v+w)(x) & =0, \\
\left(-k u+v^{\prime}\right)(x)-\frac{t^{2}}{12}\left(-k \theta+\phi^{\prime}-v^{\prime}\right)(x) & =0, \\
w(x) & =0 \\
\theta^{\prime}(x)+\nu k \phi(x) & =0, \\
-k \theta(x)+\phi^{\prime}(x)-v^{\prime}(x) & =0 .
\end{aligned}
$$

\subsubsection{Decomposition into smooth components and edge effects}

The solution of the dimensionally reduced shell model $\underline{u}$ admits the following decomposition

$$
\underline{u}=\underline{u}^{s}+\underline{u}_{t}^{b}+\underline{u}_{\sqrt{t}}^{b},
$$

where $\underline{u}^{s}$ is the smooth part, $\underline{u}^{b}$ the $t$-length scale boundary layer part and $\underline{u}_{\sqrt{t}}^{b}$ the $\sqrt{t}$-length scale boundary layer part.

This means that for the Naghdi, or R-model (2.1) of the cylindrical shell, the displacement field displays a short $t$-length boundary layer due to the shear deformation (analogous to the layer in the Reissner-Mindlin plate model), and a longer $\sqrt{t}$-length boundary layer, related to the so-called "simple edge effect". This property will ultimately decide the choice of the $h p$-FE spaces, since we want to resolve these layers.

At this point we can be more precise about these components, namely

$$
\begin{aligned}
\underline{u}^{s} & =\underline{u}^{0}+\sum_{i=5}^{8} a_{i} \underline{c}^{i} e^{\lambda_{i} x} \\
\underline{u}_{t}^{b} & =a_{9} \underline{c}^{9} e^{\lambda_{9} x}+a_{10} \underline{c}^{10} e^{\lambda_{10} x} \\
\underline{u}_{\sqrt{t}}^{b} & =\sum_{i=1}^{4} a_{i} \underline{c}^{i} e^{\lambda_{i} x} .
\end{aligned}
$$


Here

$$
\begin{aligned}
\underline{u}^{0}=\frac{f_{0}}{t^{3}} & {\left[0,-\frac{1}{k\left(1-k^{2}\right)^{2}}\left(\frac{3-\nu}{1-\nu} k^{2} t^{2}+12\right), \frac{1}{\left(1-k^{2}\right)^{2}}\left(t^{2}+\frac{24}{1-\nu}\left(\frac{t^{2}}{12} k^{2}+\frac{1-\nu}{2}\right)\right),\right.} \\
& \left.0, \frac{12}{k\left(1-k^{2}\right)}\right]
\end{aligned}
$$

is a particular solution of the Euler-Lagrange equations $(2.5), \lambda_{i}(t)$ are the eigenvalues and $\underline{c}^{i}(t)$ the corresponding eigenvectors constituting a fundamental system of (2.5). The coefficients $a_{i}$ are in each case determined by the boundary conditions.

Lemma 2.1 Let $k=2$. Then the roots of the characteristic polynomial which correspond to (2.5) admit expansions in Puiseux series with respect to the thickness variable t:

$$
\begin{aligned}
\lambda_{1} & =(1+i) \sqrt[4]{3\left(1-\nu^{2}\right)} t^{-1 / 2}+\frac{2(i-1)}{-1+\nu^{2}}\left(\sqrt[4]{3\left(1-\nu^{2}\right)}\right)^{3}\left(\frac{\nu}{24}+\frac{3}{8}\right) t^{1 / 2} \\
& +\frac{1+i}{-1+\nu^{2}} \sqrt[4]{3\left(1-\nu^{2}\right)}\left(-\frac{5}{32} \nu^{2}+\frac{1}{48} \nu-\frac{21}{32}\right) t^{3 / 2}+O\left(t^{5 / 2}\right), \\
\lambda_{2} & =-\lambda_{1}, \quad \lambda_{3}=\bar{\lambda}_{1}, \quad \lambda_{4}=-\bar{\lambda}_{1}, \\
\lambda_{5} & =(1+i) \sqrt[4]{\frac{3}{1-\nu^{2}}} t^{1 / 2}-\frac{1}{2}(i-1)\left(\frac{3}{1-\nu^{2}}\right)^{3 / 4} t^{3 / 2}+O\left(t^{5 / 2}\right), \\
\lambda_{6} & =-\lambda_{5}, \quad \lambda_{7}=\bar{\lambda}_{5}, \quad \lambda_{8}=-\bar{\lambda}_{5}, \\
\lambda_{9} & =2 \sqrt{3} t^{-1}+\frac{2 \sqrt{3}}{3} t+O\left(t^{3}\right), \\
\lambda_{10} & =-\lambda_{9} .
\end{aligned}
$$

For the proof of this lemma we refer to Appendix.

Because we assume homogeneous boundary conditions we get symmetry of the solution with respect to the axial variable $x$, and therefore the components of the displacement field $\underline{u}$ can be more compactly written as

$$
\begin{aligned}
\underline{u}^{s} & =\underline{u}^{0}+2 \operatorname{Re}\left(a_{5} \underline{c}^{5}(\cdot)\left(e^{\lambda_{5} x} \mp e^{-\lambda_{5} x}\right)\right), \\
\underline{u}_{t}^{b} & =a_{9} \underline{c}^{9}(\cdot) e^{\lambda_{9}}\left(e^{\lambda_{9} x} \mp e^{-\lambda_{9} x}\right), \\
\underline{u}_{\sqrt{t}}^{b} & =2 \operatorname{Re}\left(a_{1} \underline{c}^{1}(\cdot)\left(e^{\lambda_{1} x} \mp e^{-\lambda_{1} x}\right)\right),
\end{aligned}
$$

where the - corresponds to the components $u$ and $\theta$, (odd functions with respect to $x$ ), and + corresponds to $v, w$ and $\phi$, (even functions of $x$ ). Let us now define the coefficients

$$
M(t):=a_{5} \underline{c}^{5}(\cdot),
$$




\begin{tabular}{|c||c||c||c||c|}
\hline & & Case 1 & Case 2 & Case 3 \\
\hline \hline \multirow{5}{*}{$K$} & $u$ & 3 & $3 / 2$ & 1 \\
& $v$ & 2 & $1 / 2$ & 0 \\
& $w$ & 4 & $5 / 2$ & 2 \\
& $\theta$ & 1 & $-1 / 2$ & -1 \\
& $\phi$ & 0 & $-3 / 2$ & -2 \\
\hline \multirow{5}{*}{$L$} & $u$ & $-1 / 2$ & $-3 / 2$ & $-3 / 2$ \\
& $v$ & 0 & -1 & -1 \\
& $w$ & -1 & -2 & -2 \\
& $\theta$ & $-3 / 2$ & $-5 / 2$ & $-5 / 2$ \\
& $\phi$ & -1 & -2 & -2 \\
\hline \multirow{5}{*}{$M$} & $u$ & $-5 / 2$ & -2 & $-7 / 2$ \\
& $v$ & -3 & $-5 / 2$ & -4 \\
& $w$ & -3 & $-5 / 2$ & -4 \\
& $\theta$ & $-5 / 2$ & -2 & $-7 / 2$ \\
& $\phi$ & -3 & $-5 / 2$ & -4 \\
\hline
\end{tabular}

Table 1: Exponent $\delta$ in the asymptotics of $K(t), L(t), M(t)=O\left(t^{\delta}\right)$

$$
\begin{aligned}
K(t) & :=a_{9} \underline{c}^{9}(\cdot) e^{\lambda_{9}}, \\
L(t) & :=a_{1} \underline{c}^{1}(\cdot) e^{\lambda_{1}} .
\end{aligned}
$$

In Table 1 we present the asymptotic behaviour of these coefficients $O\left(t^{\delta}\right)$, i.e. the order $\delta$ of their expansions as Puiseux series with respect to $t$, for each of the benchmark problems and for each component of the displacement field for the dimensionally reduced shell model.

Remark 2.2 At this point we should remark that the regularity of the eigenvalues $\lambda_{i}=$ $\lambda_{i}(t ; \nu, k), i=1, . .10$, with respect to $t$, (which means their order as Puiseux expansions in $t$ ), does not depend on the Poisson ratio $\nu \in[0,1 / 2]$ and on the parameter $k$, which we assume to be a bounded integer.

This can be easily deduced from the fact that the entries of the matrix which corresponds to the two-point boundary value problem (2.5), as rational functions of $t$, have coefficients which are bounded and bounded away from 0 with respect to $\nu \in[0,1 / 2]$ and $k$. The same argument is available for the eigenvectors $\underline{c}^{i}$ and the coefficients $a_{i}$, which depend also on $t, \nu$, and $k$, but do not depend on the coordinate $x$. 


\subsubsection{Classification of Deformation states}

The deformation energy $\mathcal{E}\left(\omega_{0} ; \underline{U}\right)=\mathcal{A}_{R}\left(\omega_{0} ; \underline{U}, \underline{U}\right)=t \mathcal{A}_{m}\left(\omega_{0} ; \underline{U}, \underline{U}\right)+t^{3} \mathcal{A}_{b}\left(\omega_{0} ; \underline{U}, \underline{U}\right)$ corresponding to a subdomain $\omega_{0} \subset \omega$ consists of the membrane energy $\mathcal{E}_{m}\left(\omega_{0} ; \underline{U}\right)=t \mathcal{A}_{m}\left(\omega_{0} ; \underline{U}, \underline{U}\right)$ and of the bending energy $\mathcal{E}_{b}\left(\omega_{0} ; \underline{U}\right)=t^{3} \mathcal{A}_{b}\left(\omega_{0} ; \underline{U}, \underline{U}\right)$, where

$$
\begin{aligned}
\mathcal{A}_{m}\left(\omega_{0} ; \underline{U}, \underline{U}\right) & =\frac{1}{2} \int_{\omega_{0}}\left[\nu\left(\beta_{11}+\beta_{22}\right)^{2}+(1-\nu) \sum_{i, j=1}^{2} \beta_{i j}^{2}\right] d x d \alpha \\
& +\frac{1-\nu}{4} \int_{\omega_{0}}\left(\rho_{1}^{2}+\rho_{2}^{2}\right) d x d \alpha \\
\mathcal{A}_{b}\left(\omega_{0} ; \underline{U}, \underline{U}\right) & =\frac{1}{24} \int_{\omega_{0}}\left[\nu\left(\kappa_{11}+\kappa_{22}\right)^{2}+(1-\nu) \sum_{i, j=1}^{2} \kappa_{i j}^{2}\right] d x d \alpha
\end{aligned}
$$

A displacement field $\underline{U}$ is called membrane dominated in $\omega_{0} \subset \omega$, and the corresponding asymptotic deformation is referred to as membrane state, if its membrane energy over $\omega_{0}$ dominates asymptotically, i.e.

$$
\mathcal{E}_{b}\left(\omega_{0} ; \underline{U}\right) / \mathcal{E}\left(\omega_{0} ; \underline{U}\right) \longrightarrow 0 \text { as } t \longrightarrow 0 .
$$

Then $\underline{U}$ is called bending dominated in $\omega_{0}$, and the asymptotic deformation state is referred to as inextensional state, if the bending part of the energy over $\omega_{0}$ dominates asymptotically, i.e. if

$$
\mathcal{E}_{m}\left(\omega_{0} ; \underline{U}\right) / \mathcal{E}\left(\omega_{0} ; \underline{U}\right) \longrightarrow 0 \text { as } t \longrightarrow 0 .
$$

If $\omega_{0}=\omega$, we call the shell bending or membrane dominated.

When neither the membrane nor the bending part of the energy dominates asymptotically then we refer the corresponding states as to intermediate states. Based on the explicit form (2.2) of the solution, it can be shown [11] that asymptotically, i.e., as $t \rightarrow 0$, the deformation states are globally

Case 1: membrane dominated,

Case 2: bending dominated,

Case 3: intermediate states.

The global characterization is, however, crude in a sense, since the solution may locally, i.e. over $\omega_{0} \subset \omega$ behave differently. As an example, let us consider Case 1 , which is most interesting in applications, and the boundary-layer region $\omega_{0}=\left(1-\kappa \tilde{p} / \sqrt[4]{12\left(1-\nu^{2}\right)} \sqrt{t}, 1\right) \times(0,2 \pi)$, with $\kappa$ a constant, $\tilde{p}=(p+1) / 2$. In this case the deformation field $\underline{U}$ is globally membrane dominated, the boundary-layer component $\underline{U}^{b}$ is globally bending dominated, while the smooth component $\underline{U}^{s}$ is bending dominated in the $O(\sqrt{t})$-layer, even though it is globally membrane dominated.

Indeed, let $c:=\kappa \tilde{p} / \sqrt[4]{12\left(1-\nu^{2}\right)}$. The assertion that the smooth component $\underline{U}^{s}$ is in the layer region $\omega_{0}$ bending dominated is equivalent to $\left\|\underline{u}^{s}\right\|_{e,(1-c \sqrt{t}, 1)} \sim t^{3 / 2}\left\|\underline{u}^{s}\right\|_{1,(1-c \sqrt{t}, 1)}$, where 
by $\|\cdot\|_{e,(1-c \sqrt{t}, 1)}$ we denote the dimensionally reduced energy norm corresponding to $(1-c \sqrt{t}, 1)$. Now by the definitions of the energy norm $\|\cdot\|_{e}$ and the membrane, bending and shear strain tensors $\beta^{s}, \kappa^{s}$ and $\rho^{s}$ of $\underline{u}^{s}$ we get the following estimate:

$$
\begin{aligned}
\left\|\underline{u}^{s}\right\|_{e,(1-c \sqrt{t}, 1)} & \leq C t^{1 / 2}\left(\sum_{i, j=1}^{2}\left\|\beta_{i j}^{s}\right\|_{0,(1-c \sqrt{t}, 1)}+\sum_{i=1}^{2}\left\|\rho_{i}^{s}\right\|_{0,(1-c \sqrt{t}, 1)}\right) \\
& +C t^{3 / 2}\left(\sum_{i, j=1}^{2}\left\|\kappa_{i j}^{s}\right\|_{0,(1-c \sqrt{t}, 1)}\right) .
\end{aligned}
$$

Our assertion follows, if we show that

$$
\begin{aligned}
t^{1 / 2}\left\|\beta_{i j}^{s}\right\|_{0,(1-c \sqrt{t}, 1)} \leq C t^{3 / 2}\left\|\underline{u}^{s}\right\|_{1,(1-c \sqrt{t}, 1)}, & \forall i, j=1,2, \\
t^{1 / 2}\left\|\rho_{i}^{s}\right\|_{0,(1-c \sqrt{t}, 1)} \leq C t^{3 / 2}\left\|\underline{u}^{s}\right\|_{1,(1-c \sqrt{t}, 1)}, & \forall i=1,2 .
\end{aligned}
$$

Let us first verify this for the membrane strain tensor components $\beta_{i j}^{s}$. Indeed, we have that

$$
\begin{aligned}
t^{1 / 2}\left\|\beta_{11}^{s}\right\|_{0,(1-c \sqrt{t}, 1)} & =t^{1 / 2}\left|u^{s}\right|_{1,(1-c \sqrt{t}, 1)} \\
& =t^{1 / 2}\left\|2 \operatorname{Re}\left(M(t) \lambda_{5}(t)\left(e^{\lambda_{5}(t)(\cdot)}+e^{-\lambda_{5}(t)(\cdot)}\right)\right)\right\|_{0,(1-c \sqrt{t}, 1)} \\
& \leq C t^{1 / 2} t^{-5 / 2} t^{1 / 2} t^{1 / 4} \leq C t^{3 / 2}\left\|\underline{u}^{s}\right\|_{1,(1-c \sqrt{t}, 1)},
\end{aligned}
$$

since the $H^{1}$-norm of $\underline{u}^{s}$ in the layer region is of order $t^{-3} t^{1 / 4}$, and $\left\|e^{\lambda_{5}(t)(\cdot)} \pm e^{-\lambda_{5}(t)(\cdot)}\right\|_{0,(1-c \sqrt{t}, 1)}=$ $O\left(t^{1 / 4}\right)$ in the case with + , and $O\left(t^{3 / 4}\right)$ in the case with - . Analogously,

$$
\begin{aligned}
t^{1 / 2}\left\|\beta_{12}^{s}\right\|_{0,(1-c \sqrt{t}, 1)} & =t^{1 / 2}\left\|-k u^{s}+v^{s \prime}\right\|_{0,(1-c \sqrt{t}, 1)} \\
& =t^{1 / 2} \| 2 \operatorname{Re}\left(( - k M _ { 1 } ( t ) + \lambda _ { 5 } ( t ) M _ { 2 } ( t ) ) \left(e^{\lambda_{5}(t)(\cdot)}-\right.\right. \\
& \left.\left.-e^{-\lambda_{5}(t)(\cdot)}\right)\right) \|_{0,(1-c \sqrt{t}, 1)} \\
& \leq C t^{1 / 2} t^{-5 / 2} t^{3 / 4} \\
& \leq C t^{3 / 2}\left\|\underline{u}^{s}\right\|_{1,(1-c \sqrt{t}, 1)} .
\end{aligned}
$$

To get the desired estimate for the membrane strain tensor component $\beta_{22}^{s}$ let us first notice that

$$
\begin{aligned}
\beta_{22}^{s} & =k v^{s}+w^{s}=k v^{0}+w^{0} \\
& +2 \operatorname{Re}\left(\left(k M_{2}(t)+M_{3}(t)\right)\left(e^{\lambda_{5}(t)(\cdot)}+e^{-\lambda_{5}(t)(\cdot)}\right)\right),
\end{aligned}
$$


and $k v^{0}+w^{0}=O\left(t^{-1}\right)$. Further, making use of the homogeneous Euler-Lagrange equations corresponding to (2.5), we get that

$$
\begin{aligned}
k v^{s}+w^{s}-\left(k v^{0}+w^{0}\right) & =-\nu u^{s \prime}+\frac{1-\nu}{2}\left(-\theta^{s \prime}+w^{s \prime \prime}\right) \\
& -k \frac{t^{2}}{12}\left[-\nu k \theta^{s \prime}-k^{2}\left(\phi^{s}-\phi^{0}\right)+\frac{1-\nu}{2}\left(-k \theta^{s \prime}+\phi^{s \prime \prime}-v^{s \prime \prime}\right)\right] .
\end{aligned}
$$

Hence

$$
\begin{aligned}
t^{1 / 2}\left\|k v^{s}+w^{s}\right\|_{0,(1-c \sqrt{t}, 1)} & \leq t^{1 / 2}\left\|k v^{0}+w^{0}\right\|_{0,(1-c \sqrt{t}, 1)}+t^{1 / 2}\left\|k\left(v^{s}-v^{0}\right)+w^{s}-w^{0}\right\|_{0,(1-c \sqrt{t}, 1)} \\
& \leq C t^{1 / 2} t^{-1} t^{1 / 4}+C t^{1 / 2}\left(t^{-5 / 2} t^{1 / 2} t^{1 / 4}+t^{-2} t t^{1 / 4}\right) \\
& +C t^{1 / 2} t^{2}\left(t^{-5 / 2} t^{1 / 2} t^{1 / 4}+t^{-2} t^{1 / 4}+t^{-2} t t^{1 / 4}\right) \\
& \leq C t^{3 / 2}\left\|\underline{u}^{s}\right\|_{1,(1-c \sqrt{t}, 1)} .
\end{aligned}
$$

For the shear strain tensor components $\rho_{i}^{s}, i=1,2$, we proceed exactly as before:

$$
\begin{aligned}
t^{1 / 2}\left\|\rho_{1}^{s}\right\|_{0,(1-c \sqrt{t}, 1)} & =t^{1 / 2}\left\|-\theta^{s}+w^{s \prime}\right\|_{0,(1-c \sqrt{t}, 1)} \\
& =t^{1 / 2} \frac{2}{1-\nu}\left\|\frac{t^{2}}{12}\left(\theta^{s \prime \prime}+\nu k \phi^{s \prime}+\frac{1-\nu}{2}\left(-k^{2} \theta^{s}+k \phi^{s \prime}-k v^{s \prime}\right)\right)\right\|_{0,(1-c \sqrt{t}, 1)} \\
& \leq C t^{1 / 2} t^{2}\left(t^{-5 / 2} t t^{3 / 4}+t^{-2} t^{1 / 2} t^{3 / 4}+t^{-5 / 2} t^{3 / 4}\right) \\
& \leq t^{3 / 2}\left\|\underline{u}^{s}\right\|_{1,(1-c \sqrt{t}, 1)} \\
t^{1 / 2}\left\|\rho_{2}^{s}\right\|_{0,(1-c \sqrt{t}, 1)} & =t^{1 / 2}\left\|-\phi^{s}-k w^{s}-v^{s}\right\|_{0,(1-c \sqrt{t}, 1)} \\
& \leq t^{1 / 2}\left\|-\phi^{0}-k w^{0}-v^{0}\right\|_{0,(1-c \sqrt{t}, 1)} \\
& +t^{1 / 2}\left\|-\left(\phi^{s}-\phi^{0}\right)-k\left(w^{s}-w^{0}\right)-\left(v^{s}-v^{0}\right)\right\|_{0,(1-c \sqrt{t}, 1)} \\
& \leq C t^{1 / 2} t^{-1} t^{1 / 4} \\
& +C t^{1 / 2} t^{2}\left\|-\nu k \theta^{s \prime}-k^{2}\left(\phi^{s}-\phi^{0}\right)+\frac{1-\nu}{2}\left(-k \theta^{s \prime}+\phi^{s \prime \prime}-v^{s \prime \prime}\right)\right\|_{0,(1-c \sqrt{t}, 1)} \\
& \leq C t^{3 / 2}\left\|\underline{u}^{s}\right\|_{1,(1-c \sqrt{t}, 1)} .
\end{aligned}
$$

\section{$2.4 h p$-Finite Element Method. Main result.}

In each of the three benchmark problems we will prove the existence of a FE approximation $\tilde{U}^{h p}$ of the exact solution of our minimization problem, with respect to a particular grid of the parametrization domain $\omega$, such that the relative error in the energy norm ||$\cdot|| \mid$ decays exponentially with respect to the polynomial degree $p$.

The $h p$-FE spaces $\underline{\mathcal{U}}^{h p} \subset \underline{\mathcal{U}}$ consist of all kinematically admissible displacements $\underline{U} \in \underline{\mathcal{U}}$ which are tensor product polynomials of degree at most $2 p$ in $(x, \alpha)$ on each element of a special 


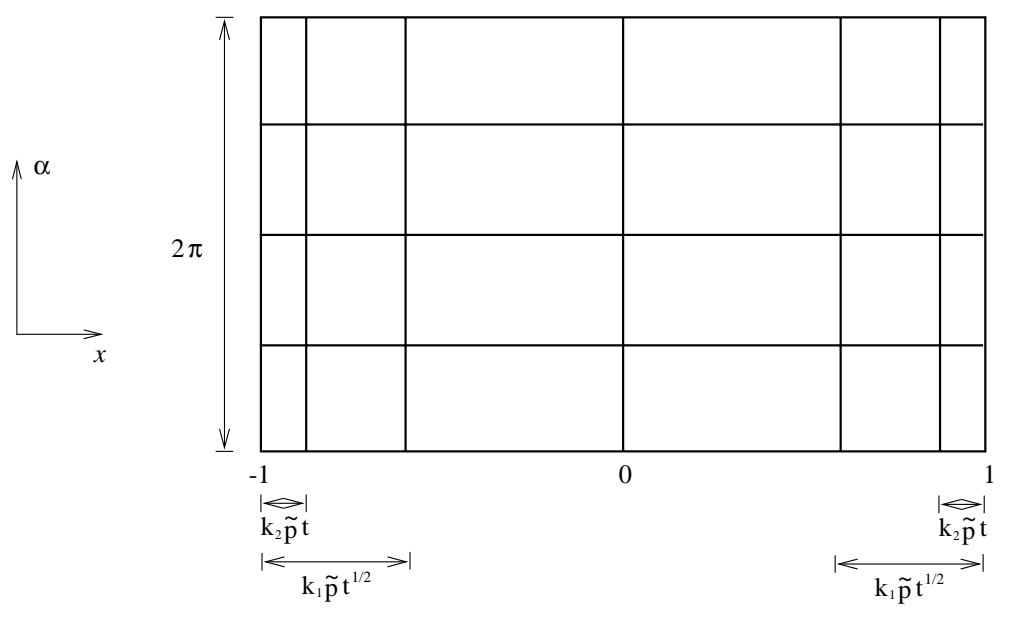

Figure 1: The triangulation $\mathcal{T}$ of the domain $\omega$.

triangulation $\mathcal{T}$ of the domain $\omega$ shown in Figure 1. The FE-solution minimizes the energy functional over the $h p$-FE space $\underline{\mathcal{U}}^{h p}$ :

$$
\underline{\tilde{U}}^{h p}=\arg \left\{\min _{\underline{V} \in \underline{\mathcal{U}}^{h p}} \mathcal{F}_{R}(\underline{V})\right\},
$$

and the relative error satisfies

$$
\frac{\left\|\underline{U}-\underline{\tilde{U}}^{h p}|\||\right.}{\|\underline{U} \mid\|} \leq C t^{\beta} e^{-\eta p},
$$

where $C, \eta>0$ are positive constants independent of the thickness $t$ of the shell, $-1 \leq \beta \leq 0$ is a locking factor, and $p$ denotes the polynomial degree of the discrete approximation ${ }^{3}$.

Theorem 2.3 There hold the following convergence estimates:

$$
\begin{array}{rlrl}
\frac{\left\|\underline{U}-\underline{\tilde{U}}^{h p} \mid\right\|}{\|\underline{U}\|} & \leq C t^{-3 / 4} e^{-\eta p}, & & \text { in the first case, and } \\
& \leq C t^{-1} e^{-\eta p}, \quad & \text { in the last two cases. }
\end{array}
$$

Corollary 2.4 Let $\mathcal{T}_{1}$ be the mesh obtained from the mesh $\mathcal{T}$ in Figure 1 by bisecting the elements along a diagonal into two triangles, and let $\mathcal{V}^{\text {hp }}$ be the space of continuous, piecewise polynomials of degree $p$ on $\mathcal{T}_{1}$, subject to the kinematical constraints. Then the FEM based on $\mathcal{V}^{\text {hp }}$ satisfies the same error estimates as in Theorem 2.3, with $\eta$ replaced with $\eta / 2$.

\footnotetext{
${ }^{3}$ We assume here the absence of quadrature errors; this is realistic, since in the case of cylindrical shells all curvature terms are constant, i.e. all integrands in the bilinear form are polynomial.
} 
Proof. Since $\mathcal{V}^{h p^{\prime}} \supset \mathcal{U}^{h p}$ with $p^{\prime}=2 p$, the corollary follows.

Remark 2.5 In our convergence analysis below, we prove in fact more detailed error estimates for each of the solution components $\underline{u}^{s}, \underline{u}_{t}^{b}, \underline{u}_{\sqrt{t}}^{b}$; they indicate that even in the (membrane dominated) Case 1 we encounter membrane locking due to the boundary layer $\underline{u}_{\sqrt{t}}^{b}$ (which unlike the overall problem is bending dominated). In each case, the $\sqrt{t}$-layer is dominant and causes the membrane locking. It is therefore essential in practice to resolve this length scale. In contrast, the $t$-layer $\underline{u}_{t}^{b}$ caused by the shear deformation does not contribute to the energy convergence; it does, however, influence pointwise stresses near $\partial \omega$.

\section{Proof of Theorem 2.3}

\subsection{Definition of the $h p$-Interpolant $\underline{U}^{h p}$}

Because of the product structure of the exact solution $\underline{U}(x, \alpha)=\underline{u}(x) \otimes \underline{v}(\alpha)$, we will look for an approximative solution with separated variables

$$
\underline{U}^{h p}(x, \alpha)=\underline{u}^{h p}(x) \otimes \underline{v}^{p}(\alpha), \quad(x, \alpha) \in(-1,1) \times(0,2 \pi) .
$$

In what follows $\underline{v}^{p}$ will be a piecewise polynomial of degree $p$ on a uniform mesh on $[0,2 \pi]$, interpolating $\underline{v}=(\cos k \alpha, \sin k \alpha, \cos k \alpha, \cos k \alpha, \sin k \alpha)$, an entire function of $\alpha$, on each interval in the mapped Chebyshev points. We have that

$$
\left\|\underline{v}-\underline{v}^{p}\right\|_{1,[0,2 \pi]} \leq C_{\eta_{0}} e^{-\eta_{0} p}, \quad \forall \eta_{0}>0, p=1,2,3, \ldots
$$

where the constants $C_{\eta_{0}}, \eta_{0}>0$ do not depend on the polynomial degree $p$, the thickness variable $t$, or on the parameter $k$, as long as $k$ remains bounded as in our hypothesis.

Then we still need to construct $\underline{u}^{h p}$; we will obtain it in the form

$$
\underline{u}^{h p}=\underline{u}^{s, h p}+\underline{u}_{t}^{b, h p}+\underline{u}_{\sqrt{t}}^{b, h p},
$$

where

$$
\begin{aligned}
\underline{u}^{s, h p} & =\underline{u}^{0}+2 \operatorname{Re}\left(M(t)\left(s_{1} \mp \tilde{s}_{1}\right)\right), \\
\underline{u}_{t}^{b, h p} & =K(t)\left(v_{t} \mp \tilde{v}_{t}\right), \\
\underline{u}_{\sqrt{t}}^{b, h p} & =2 \operatorname{Re}\left(L(t)\left(v_{\sqrt{t}} \mp \tilde{v}_{\sqrt{t}}\right)\right) .
\end{aligned}
$$

To define the $h p$-Interpolant we still have to explain the choice of $s_{1}, \tilde{s}_{1}, v_{t}, \tilde{v}_{t}, v_{\sqrt{t}}, \tilde{v}_{\sqrt{t}}$. We start with the approximations $s_{1}$ and $\tilde{s}_{1}$ of the smooth part. 
As we have seen, the expansions in $t$ of $\lambda_{i}=\lambda_{i}(t), i=5, . ., 8$ consist only of rational, positive powers of the thickness parameter $t \in\left[0, t_{0}\right]$ in a bounded, closed interval. This means that the domains of analyticity of the exponential functions $e^{\lambda_{5}(t) x}, e^{-\lambda_{5}(t) x}$, analytic with respect to the axial variable $x$, do not depend on $t \in\left[0, t_{0}\right]$ and $\nu \in[0,1 / 2]$. Therefore we can approximate them uniformly with respect to $t \in\left[0, t_{0}\right]$ with polynomials of degree $p$, which interpolate them in the Chebyshev points, such that the absolute error in the $H^{1}$-norm is

$$
\begin{aligned}
\left\|e^{\lambda_{5}(t)(\cdot)}-s_{1}(\cdot)\right\|_{1,(-1,1)} & \leq C e^{-\eta_{1} p} \\
\left\|e^{-\lambda_{5}(t)(\cdot)}-\tilde{s}_{1}(\cdot)\right\|_{1,(-1,1)} & \leq C e^{-\eta_{1} p}
\end{aligned}
$$

for some positive constants $C, \eta_{1}>0$, which do not depend on $t \in\left[0, t_{0}\right]$ and the Poisson ratio $\nu \in[0,1 / 2]$.

The $t$-length scale boundary layer component has the form

$$
\underline{u}_{t}^{b}=K(t)\left(e^{-\lambda_{9}(t)(1-x)} \mp e^{-\lambda_{9}(t)(1-x)}\right)
$$

and we split

$$
\lambda_{9}(t)=\frac{\sqrt{12}}{t}+\lambda_{0}(t)
$$

where the principal part

$$
\lambda_{0}(t)=\frac{\sqrt{12}}{3} t+O\left(t^{3}\right)
$$

is analytic with respect to $t \in\left[0, t_{0}\right]$. Then we have the following decomposition

$$
e^{-\lambda_{9}(t)(1 \pm x)}=u_{1 \pm} \cdot u_{2 \pm},
$$

into a boundary-layer and a smooth part, respectively, given by

$$
u_{1 \pm}:=e^{(-\sqrt{12} / t)(1 \pm x)}, \quad u_{2 \pm}:=e^{-\lambda_{0}(t)(1 \pm x)} .
$$

We define now the $h p$-interpolants in analogous fashion, i.e.

$$
v_{t}:=u_{1+}^{h p} \cdot u_{2+}^{p}, \quad \tilde{v}_{t}:=u_{1-}^{h p} \cdot u_{2-}^{p},
$$

where $u_{1 \pm}^{h p}$ are $h p$-interpolants of the boundary-layer functions $u_{1 \pm}=e^{(-\sqrt{12} / t)(1 \pm x)}$ with respect to a three-element mesh

$$
\mathcal{T}_{0}=\left\{-1,-1+\frac{\kappa \tilde{p} t}{\sqrt{12}}, 1-\frac{\kappa \tilde{p} t}{\sqrt{12}}, 1\right\}
$$

The two small elements at the ends of the length interval $(-1,1)$ are of order $O(p t)$, since $\tilde{p}=$ $p+1 / 2$. The robust exponential rate of convergence for these boundary-layer approximations is given in [14]. For the convenience of the reader we will restate this result here: 
Lemma 3.1 Let $u_{\lambda, \varepsilon}(x)=\exp (-\lambda(x+1) / \varepsilon), x \in(-1,1)$ with $\varepsilon>0, \lambda=a+i b, a^{2}+b^{2}=1$. Let $(\mathcal{T}, p)$ be such that for some $\kappa$ independent of $p$, $\varepsilon$ satisfying $0<\kappa_{0} \leq \kappa<4 / e$,

$$
\begin{aligned}
& \vec{p}=\{p, 1\}, \quad \mathcal{T}=\{-1,-1+\kappa \tilde{p} \varepsilon, 1\} \quad \text { if } \kappa \tilde{p} \varepsilon<2 \text {, } \\
& \vec{p}=\{p\}, \quad \mathcal{T}=\{-1,1\} \quad \text { if } \kappa \tilde{p} \varepsilon \geq 2 \text {. }
\end{aligned}
$$

Then there exists $u_{p} \in\left\{u \in C^{0}(-1,1):\left.u\right|_{I_{j}} \in \mathcal{P}_{p(j)}\left(I_{j}\right), I_{j} \in \mathcal{T}\right\}$, satisfying

$$
u_{p}( \pm 1)=u_{\lambda, \varepsilon}( \pm 1)
$$

and the error estimate

$$
\begin{aligned}
\left\|u_{\lambda, \varepsilon}-u_{p}\right\|_{0} & \leq C \varepsilon^{1 / 2} q^{\tilde{p}}, \\
\left|u_{\lambda, \varepsilon}-u_{p}\right|_{1} & \leq C \varepsilon^{-1 / 2} q^{\tilde{p}} .
\end{aligned}
$$

Here, the constants are independent of $p$ and $\varepsilon$ but depend on $\kappa_{0}$ and $q<1$ is given by

$$
q:= \begin{cases}e / 2 \tilde{p} \varepsilon & \text { if } \kappa \tilde{p} \varepsilon \geq 2 \\ \max \left\{\kappa e / 4, e^{-a(\kappa-\delta)}\right\} & \text { otherwise }\end{cases}
$$

with $\delta>(\ln \tilde{p}) / 2 \tilde{p}$ arbitrary.

With the same arguments that we used to approximate the smooth components $e^{ \pm \lambda_{5}(t) x}$, let us take $u_{2 \pm}^{p}$ as the interpolants of the components $u_{2 \pm}$. Then we have that for some positive constants $C$ and $\eta_{1}>0$ :

$$
\left\|u_{2 \pm}-u_{2 \pm}^{p}\right\|_{1,(-1,1)} \leq C e^{-\eta_{1} p} .
$$

Now, with our special choice of the mesh and of the FE interpolants $u_{1 \pm}^{h p}$,

$$
\begin{aligned}
\left\|u_{1 \pm}-u_{1 \pm}^{h p}\right\|_{0,(-1,1)} & \leq C t^{1 / 2} e^{-\eta_{2} p} \\
\left|u_{1 \pm}-u_{1 \pm}^{h p}\right|_{1,(-1,1)} & \leq C t^{-1 / 2} e^{-\eta_{2} p}
\end{aligned}
$$

for some $C, \eta_{2}>0$.

Analogously, let us next define $v_{\sqrt{t}}$ and $\tilde{v}_{\sqrt{t}}$. We separate as before the expansion with respect to $t$ of the first eigenvalue $\lambda_{1}(t)$ in its singular and principal parts

$$
\begin{aligned}
\lambda_{1}(t) & =(1+i) \sqrt[4]{3\left(1-\nu^{2}\right)} t^{-1 / 2}+\tilde{\lambda}_{0}(t) \\
\tilde{\lambda}_{0}(t) & =\frac{2(i-1)}{-1+\nu^{2}}\left(\sqrt[4]{3\left(1-\nu^{2}\right)}\right)^{3}\left(\frac{\nu}{24}+\frac{3}{8}\right) t^{1 / 2} \\
& +\frac{1+i}{-1+\nu^{2}} \sqrt[4]{3\left(1-\nu^{2}\right)}\left(-\frac{5}{32} \nu^{2}+\frac{1}{48} \nu-\frac{21}{32}\right) t^{3 / 2}+O\left(t^{5 / 2}\right)
\end{aligned}
$$


and notice that the coefficient of the singular part has its real part bounded and bounded away from 0 :

$$
\operatorname{Re}\left[(1+i) \sqrt[4]{3\left(1-\nu^{2}\right)}\right]>0
$$

uniformly with respect to $\nu \in[0,1 / 2]$. Then the boundary-layer function is correspondingly splitted in

$$
e^{-\lambda_{1}(t)(1 \pm x)}=u_{1 \pm} u_{2 \pm}
$$

where now

$$
u_{1 \pm}:=e^{-(1+i) \sqrt[4]{3\left(1-\nu^{2}\right)} t^{-1 / 2}(1 \pm x)}, \quad u_{2 \pm}:=e^{-\tilde{\lambda}_{0}(t)(1 \pm x)} .
$$

Then, as before, Lemma 3.1 implies the use of a three-element mesh, such that the two small elements at the ends of the interval $(-1,1)$ are now of length $O(p \sqrt{t})$,

$$
\mathcal{T}_{1}=\left\{-1,-1+\frac{\kappa \tilde{p} \sqrt{t}}{\sqrt[4]{12\left(1-\nu^{2}\right)}}, 1-\frac{\kappa \tilde{p} \sqrt{t}}{\sqrt[4]{12\left(1-\nu^{2}\right)}}, 1\right\}
$$

and of $h p$-interpolants $u_{1 \pm}^{h p}$ of $u_{1 \pm}$ with respect to this mesh, such that

$$
\begin{aligned}
\left\|u_{1 \pm}-u_{1 \pm}^{h p}\right\|_{0,(-1,1)} & \leq C t^{1 / 4} e^{-\eta_{2} p} \\
\left|u_{1 \pm}-u_{1 \pm}^{h p}\right|_{1,(-1,1)} & \leq C t^{-1 / 4} e^{-\eta_{2} p}
\end{aligned}
$$

for some positive constants $C, \eta_{2}>0$, which do not depend on $\nu \in[0,1 / 2], t \in\left(0, t_{0}\right]$ and the polynomial degree $p$. Then take $v_{\sqrt{t}}:=u_{1+}^{h p} \cdot u_{2+}^{p}, \tilde{v}_{\sqrt{t}}:=u_{1-}^{h p} \cdot u_{2-}^{p}$, with $u_{1 \pm}^{h p}$ specified before, and $u_{2 \pm}^{p}$ as interpolants of $u_{2 \pm}$ in the mapped Chebychev points on each element of $\mathcal{T}_{1}$, such that the absolute error in the $H^{1}$-norm is bounded by :

$$
\left\|u_{2 \pm}-u_{2 \pm}^{p}\right\|_{1,(-1,1)} \leq C e^{-\eta_{1} p} .
$$

\subsection{Energy norm error estimates}

The relative error in the shell energy norm can be estimated in terms of the relative error of the smooth and boundary-layer components of the displacement field as follows:

$$
\frac{\left\|\underline{U}-\underline{U}^{h p} \mid\right\|}{\|\underline{U}\|} \leq A_{s} \frac{\left\|\underline{U}^{s}-\underline{U}^{s, h p}\right\| \mid}{\left\|\underline{U}^{s} \mid\right\|}+A_{b} \frac{\left\|\underline{U}^{b}-\underline{U}^{b, h p}\right\| \mid}{\left\|\underline{U}^{b}\right\| \mid},
$$

where the amplitudes $A_{s}, A_{b}$ are given by

$$
A_{s}=\frac{\left\|\left|\underline{U}^{s}\right|\right\|}{\|\underline{U}\|} \sim 1, \quad A_{b}=\frac{\left\|\underline{U}^{b}\right\|}{\|\underline{U} \mid\|} \sim t^{1 / 4}
$$


in Cases 1, 2, and

$$
A_{s} \sim t^{1 / 4}, \quad A_{b} \sim 1,
$$

in Case 3. With our definitions,

$$
\begin{aligned}
\underline{U}^{s} & =\underline{u}^{s} \otimes \underline{v}, & \underline{U}^{s, h p} & =\underline{u}^{s, h p} \otimes \underline{v}^{p}, \\
\underline{U}^{b} & =\underline{u}^{b} \otimes \underline{v}, & \underline{U}^{b, h p} & =\underline{u}^{b, h p} \otimes \underline{v}^{p}, \\
\underline{u}^{b} & =\underline{u}_{t}^{b}+\underline{u}_{\sqrt{t}}^{b}, & \underline{u}^{b, h p} & =\underline{u}^{b, h p}+\underline{u}_{\sqrt{t}}^{b, h p},
\end{aligned}
$$

where $\underline{u}^{s, h p}, \underline{u}_{t}^{b, h p}$ and $\underline{u}_{\sqrt{t}}^{b, h p}$ are defined in (3.2).

Lemma 3.2 For all $\eta_{0}>0$ there exist positive constants $C, C_{\eta_{0}}$, such that the absolute errors in the energy norm $\|\cdot \mid\|$ of the smooth and boundary-layer parts can be estimated as follows

$$
\begin{aligned}
\left\|\underline{U}^{s}-\underline{U}^{s, h p}\right\| & \leq C t^{1 / 2}\left[C_{\eta_{0}}\left\|\underline{u}^{s}-\underline{u}^{s, h p}\right\|_{1,(-1,1)} e^{-\eta_{0} p}+\left\|\underline{u}^{s}-\underline{u}^{s, h p}\right\|_{1,(-1,1)}\right. \\
& \left.+C_{\eta_{0}}\left\|\underline{u}^{s}\right\|_{1,(-1,1)} e^{-\eta_{0} p}\right] \\
\left\|\underline{U}^{b}-\underline{U}^{b, h p}\right\| & \leq C t^{1 / 2}\left[C_{\eta_{0}}\left\|\underline{u}^{b}-\underline{u}^{b, h p}\right\|_{1,(-1,1)} e^{-\eta_{0} p}+\left\|\underline{u}^{b}-\underline{u}^{b, h p}\right\|_{1,(-1,1)}\right. \\
& \left.+C_{\eta_{0}}\left\|\underline{u}^{b}\right\|_{1,(-1,1)} e^{-\eta_{0} p}\right] .
\end{aligned}
$$

Proof. Let us observe that the definitions of the $h p$-FEM interpolants and simple triangle inequalities imply that

$$
\begin{aligned}
\left\|\underline{U}^{s}-\underline{U}^{s, h p}\right\| & \leq C t^{1 / 2}\left\|\underline{U}^{s}-\underline{U}^{s, h p}\right\|_{1, \omega}=C t^{1 / 2}\left\|\underline{u}^{s} \otimes \underline{v}-\underline{u}^{s, h p} \otimes \underline{v}^{p}\right\|_{1, \omega} \\
& \leq C t^{1 / 2}\left[\left\|\underline{u}^{s}-\underline{u}^{s, h p}\right\|_{1,(-1,1)}\left\|\underline{v}^{p}\right\|_{1,(0,2 \pi)}+\left\|\underline{u}^{s}\right\|_{1,(-1,1)}\left\|\underline{v}-\underline{v}^{p}\right\|_{1,(0,2 \pi)}\right] \\
& \leq C t^{1 / 2}\left[\left\|\underline{u}^{s}-\underline{u}^{s, h p}\right\|_{1,(-1,1)}\left\|\underline{v}-\underline{v}^{p}\right\|_{1,(0,2 \pi)}\right. \\
& \left.+\left\|\underline{u}^{s}-\underline{u}^{s, h p}\right\|_{1,(-1,1)}\|\underline{v}\|_{1,(0,2 \pi)}+\left\|\underline{u}^{s}\right\|_{1,(-1,1)}\left\|\underline{v}-\underline{v}^{p}\right\|_{1,(0,2 \pi)}\right],
\end{aligned}
$$

and in exactly the same way we find

$$
\begin{aligned}
\left\|\underline{U}^{b}-\underline{U}^{b, h p}\right\| \mid & \leq C t^{1 / 2}\left[\left\|\underline{u}^{b}-\underline{u}^{b, h p}\right\|_{1,(-1,1)}\left\|\underline{v}-\underline{v}^{p}\right\|_{1,(0,2 \pi)}\right. \\
& \left.+\left\|\underline{u}^{b}-\underline{u}^{b, h p}\right\|_{1,(-1,1)}\|\underline{v}\|_{1,(0,2 \pi)}+\left\|\underline{u}^{b}\right\|_{1,(-1,1)}\left\|\underline{v}-\underline{v}^{p}\right\|_{1,(0,2 \pi)}\right] .
\end{aligned}
$$

The proof of the lemma follows now easily because of the estimates

$$
\left\|\underline{v}-\underline{v}^{p}\right\|_{1,(0,2 \pi)} \leq C_{\eta_{0}} e^{-\eta_{0} p}
$$


and

$$
\|\underline{v}\|_{1,(0,2 \pi)} \leq C
$$

Moreover, we notice that up to some constants which are independent of $t$, but depend on the parameter $k$ the following equivalences hold:

$$
\left\|\underline { U } ^ { s } \left|\|\sim\| \underline{u}^{s}\left\|_{e}, \quad\right\| \underline{U}^{b}\|\mid \sim\| \underline{u}^{b} \|_{e} .\right.\right.
$$

Therefore the $h p$-approximation problem is reduced to a one-dimensional one.

Lemma 3.3 There exist constants $C$ and $\eta_{1}>0$ independent of $t \in\left(0, t_{0}\right], \nu \in\left[0, \frac{1}{2}\right]$ and the polynomial degree $p$, such that $\left\|\underline{u}^{s}-\underline{u}^{s, h p}\right\|_{1,(-1,1)} \leq C\|M(t)\| e^{-\eta_{1} p}$. Here we denote by $\|M(t)\|$ the $l^{2}$-norm in $\mathbb{R}^{5}$ of the vector-valued coefficient $M(t)$.

Proof. Indeed, taking into account the definitions of $\left\|\underline{u}^{s}\right\|$ and $\left\|\underline{u}^{s, h p}\right\|$

$$
\underline{u}^{s}=\underline{u}^{0}+2 \operatorname{Re}\left(M(t)\left(e^{\lambda_{5}(t)(\cdot)} \mp e^{-\lambda_{5}(t)(\cdot)}\right), \quad \underline{u}^{s, h p}=\underline{u}^{0}+2 \operatorname{Re}\left(M(t)\left(s_{1} \mp \tilde{s_{1}}\right)\right),\right.
$$

we find that

$$
\begin{aligned}
\left\|\underline{u}^{s}-\underline{u}^{s, h p}\right\|_{1,(-1,1)} & \leq 2\|M(t)\|\left(\left\|e^{\lambda_{5}(t)(\cdot)}-s_{1}(t, \cdot)\right\|_{1,(-1,1)}+\left\|e^{-\lambda_{5}(t)(\cdot)}-\tilde{s}_{1}(t, \cdot)\right\|_{1,(-1,1)}\right) \\
& \leq C\|M(t)\| e^{-\eta_{1} p}
\end{aligned}
$$

with constants $C$ and $\eta_{1}$ independent of $t \in\left(0, t_{0}\right], \nu \in\left[0, \frac{1}{2}\right]$ and the polynomial degree $p$.

Lemma 3.4 There exist constants $C$ and $\beta>0$, independent of $t \in\left(0, t_{0}\right], \nu \in\left[0, \frac{1}{2}\right]$ and the polynomial degree $p$, such that

$$
\left\|\underline{u}^{b}-\underline{u}^{b, h p}\right\|_{1,(-1,1)} \leq C\left(\|K(t)\| t^{-1 / 2}+\|L(t)\| t^{-1 / 4}\right) e^{-\beta p} .
$$

Proof. Let us first recall that

$$
\begin{aligned}
\underline{u}^{b}= & \underline{u}_{t}^{b}+\underline{u}_{\sqrt{t}}^{b}=K(t)\left(e^{-\lambda_{9}(t)(1-\cdot)} \mp e^{-\lambda_{9}(t)(1+\cdot)}\right)+ \\
& 2 \operatorname{Re}\left(L(t)\left(e^{-\lambda_{1}(t)(1-\cdot)} \mp e^{-\lambda_{1}(t)(1+\cdot)}\right)\right), \\
\underline{u}^{b, h p}= & \underline{u}_{t}^{b, h p}+\underline{u}_{\sqrt{t}}^{b, h p}=K(t)\left(v_{t} \mp \tilde{v}_{t}\right)+2 \operatorname{Re}\left(L(t)\left(v_{\sqrt{t}} \mp \tilde{v}_{\sqrt{t}}\right)\right) .
\end{aligned}
$$


This implies the following estimate for the absolute error of the boundary-layer component in the $H^{1}$-norm

$$
\begin{aligned}
\left\|\underline{u}^{b}-\underline{u}^{b, h p}\right\|_{1,(-1,1)} & \leq\left\|\underline{u}_{t}^{b}-\underline{u}_{t}^{b, h p}\right\|_{1,(-1,1)}+\left\|\underline{u}_{\sqrt{t}}^{b}-\underline{u}_{\sqrt{t}}^{b, h p}\right\|_{1,(-1,1)} \\
& \leq\|K(t)\|\left(\left\|e^{-\lambda_{9}(t)(1-\cdot)}-v_{t}\right\|_{1,(-1,1)}+\left\|e^{-\lambda_{9}(t)(1+\cdot)}-\tilde{v}_{t}\right\|_{1,(-1,1)}\right) \\
& +2\|L(t)\|\left(\left\|e^{-\lambda_{1}(t)(1-\cdot)}-v_{\sqrt{t}}\right\|_{1,(-1,1)}+\left\|e^{-\lambda_{1}(t)(1+\cdot)}-\tilde{v}_{\sqrt{t}}\right\|_{1,(-1,1)}\right) .
\end{aligned}
$$

To complete the proof we need the following

Lemma 3.5 For $j=0,1$ and some positive constants $C$ and $\beta>0$, independent of $t \in$ $\left(0, t_{0}\right], \nu \in[0,1 / 2]$ and the polynomial degree $p$, the following estimates hold:

$$
\begin{aligned}
\left\|e^{-\lambda_{9}(t)(1-\cdot)}-v_{t}\right\|_{j,(-1,1)} & \leq C t^{1 / 2-j} e^{-\beta p} \\
\left\|e^{-\lambda_{9}(t)(1+\cdot)}-\tilde{v}_{t}\right\|_{j,(-1,1)} & \leq C t^{1 / 2-j} e^{-\beta p} \\
\left\|e^{-\lambda_{1}(t)(1-\cdot)}-v_{\sqrt{t}}\right\|_{j,(-1,1)} & \leq C t^{1 / 4-j / 2} e^{-\beta p} \\
\left\|e^{-\lambda_{1}(t)(1+\cdot)}-\tilde{v}_{\sqrt{t}}\right\|_{j,(-1,1)} & \leq C t^{1 / 4-j / 2} e^{-\beta p}
\end{aligned}
$$

These estimates and (3.9) conclude now the proof of Lemma 3.4.

Proof of Lemma 3.5. We will prove only the last two estimates, the others can be deduced analogously. Recall that we splitted:

$$
\begin{aligned}
\lambda_{1}(t) & =(1+i) \sqrt[4]{3\left(1-\nu^{2}\right)} t^{-1 / 2}+\tilde{\lambda}_{0}(t) \\
\tilde{\lambda}_{0}(t) & =\frac{2(i-1)}{-1+\nu^{2}}\left(\sqrt[4]{3\left(1-\nu^{2}\right)}\right)^{3}\left(\frac{\nu}{24}+\frac{3}{8}\right) t^{1 / 2} \\
& +\frac{1+i}{-1+\nu^{2}} \sqrt[4]{3\left(1-\nu^{2}\right)}\left(-\frac{5}{32} \nu^{2}+\frac{1}{48} \nu-\frac{21}{32}\right) t^{3 / 2}+O\left(t^{5 / 2}\right),
\end{aligned}
$$

which means that

$$
e^{-\lambda_{1}(t)(1+x)}=e^{-(1+i) \sqrt[4]{3\left(1-\nu^{2}\right)} t^{-1 / 2}(1+x)} e^{-\tilde{\lambda}_{0}(t)(1+x)}
$$

Let us now define

$$
\begin{aligned}
& u_{1}:=e^{-(1+i) \sqrt[4]{3\left(1-\nu^{2}\right)} t^{-1 / 2}(1+x)}, \\
& u_{2}:=e^{-\tilde{\lambda}_{0}(t)(1+x)}
\end{aligned}
$$


and observe that $\operatorname{Re}\left((1+i) \sqrt[4]{3\left(1-\nu^{2}\right)}\right)>0$ is bounded and bounded away from 0 uniformly with respect to $\nu \in[0,1 / 2]$.

Then, Lemma 3.1 implies that there exist $u_{1}^{h p}( \pm 1)=u_{1}( \pm 1)$, piecewise polynomials of degree $p$ on the mesh

$$
\left\{-1,-1+\frac{\kappa \tilde{p} \sqrt{t}}{\sqrt[4]{12\left(1-\nu^{2}\right)}}, 1-\frac{\kappa \tilde{p} \sqrt{t}}{\sqrt[4]{12\left(1-\nu^{2}\right)}}, 1\right\}
$$

such that

$$
\left\|u_{1}-u_{1}^{h p}\right\|_{0,(-1,1)} \leq C t^{1 / 4} e^{-\eta_{2} p}
$$

and

$$
\left|u_{1}-u_{1}^{h p}\right|_{1,(-1,1)} \leq C t^{-1 / 4} e^{-\eta_{2} p},
$$

for some constants $C$ and $\eta_{2}>0$ independent of $t \in\left(0, t_{0}\right], p$ and $\nu \in[0,1 / 2]$. Here again $\tilde{p}=(p+1) / 2$.

As explained in the previous section, the smooth component $u_{2}$ can be approximated by a piecewise polynomial with respect to $x, u_{2}^{p} \in \mathcal{P}_{p}([-1,1])$, interpolating $u_{2}$ at the end points, $u_{2}^{p}( \pm 1)=u_{2}( \pm 1)$, such that the absolute error in the $H^{1}$-norm has an exponential rate of convergence

$$
\left\|u_{2}-u_{2}^{p}\right\|_{1,(-1,1)} \leq C e^{-\eta_{1} p},
$$

for some positive constants $C$ and $\eta_{1}$ independent of $t \in\left(0, t_{0}\right], p$ and $\nu \in[0,1 / 2]$. This implies immediately that

$$
\begin{aligned}
\left\|e^{-\lambda_{1}(t)(1+x)}-u_{1}^{h p} u_{2}^{p}\right\|_{0,(-1,1)} & =\left\|u_{1} u_{2}-u_{1}^{h p} u_{2}^{p}\right\|_{0,(-1,1)} \\
& \leq\left\|u_{1}-u_{1}^{h p}\right\|_{0,(-1,1)}\left\|u_{2}\right\|_{\infty}+\left\|u_{1}^{h p}\right\|_{0,(-1,1)}\left\|u_{2}-u_{2}^{p}\right\|_{\infty} \\
& \leq C t^{1 / 4} e^{-\eta_{2} p}+C t^{1 / 4} e^{-\eta_{1} p} \\
& \leq C t^{1 / 4} e^{-\beta p}
\end{aligned}
$$

with $\beta:=\min \left\{\eta_{1}, \eta_{2}\right\}>0$ and $C$ a positive constant.

In order to deduce these estimates, let us first notice that

$$
\begin{aligned}
\left\|u_{1}\right\|_{0,(-1,1)} & =\left(\int_{-1}^{1}\left|u_{1}(x)\right|^{2} d x\right)^{1 / 2}=\left[\int_{-1}^{1} e^{-2 \sqrt[4]{3\left(1-\nu^{2}\right)} t^{-1 / 2}(1+x)} d x\right]^{1 / 2} \\
& =\left[\frac{1-e^{-4} \sqrt[4]{3\left(1-\nu^{2}\right)} t^{-1 / 2}}{2 \sqrt[4]{3\left(1-\nu^{2}\right)} t^{-1 / 2}}\right]^{1 / 2} \leq C t^{1 / 4}
\end{aligned}
$$

and because of the triangle inequality it follows easily that

$$
\begin{aligned}
\left\|u_{1}^{h p}\right\|_{0,(-1,1)} & \leq\left\|u_{1}-u_{1}^{h p}\right\|_{0,(-1,1)}+\left\|u_{1}\right\|_{0,(-1,1)} \\
& \leq C t^{1 / 4} e^{-\eta_{2} p}+C t^{1 / 4} \leq C t^{1 / 4} .
\end{aligned}
$$


In order to get an estimate for the absolute error of the $H^{1}$-seminorm we proceed analogously:

$$
\begin{aligned}
\left|e^{-\lambda_{1}(t)(1+x)}-u_{1}^{h p} u_{2}^{p}\right|_{1,(-1,1)} & =\left\|\partial_{x}\left(u_{1} u_{2}\right)-\partial_{x}\left(u_{1}^{h p} u_{2}^{p}\right)\right\|_{0,(-1,1)} \\
& =\left\|\dot{u}_{1} u_{2}+u_{1} \dot{u}_{2}-\dot{u}_{1}^{h p} u_{2}^{p}-u_{1}^{h p} \dot{u}_{2}^{p}\right\|_{0,(-1,1)} \\
& \leq\left\|\dot{u}_{1}-\dot{u}_{1}^{h p}\right\|_{0,(-1,1)}\left\|u_{2}\right\|_{\infty}+\left\|\dot{u}_{1}^{h p}\right\|_{0,(-1,1)}\left\|u_{2}-u_{2}^{p}\right\|_{\infty} \\
& +\left\|u_{1}-u_{1}^{h p}\right\|_{0,(-1,1)}\left\|\dot{u}_{2}\right\|_{\infty}+\left\|u_{1}^{h p}\right\|_{0,(-1,1)}\left\|\dot{u}_{2}-\dot{u}_{2}^{p}\right\|_{\infty} \\
& \leq C\left(t^{-1 / 4} e^{-\eta_{2} p}+t^{-1 / 4} e^{-\eta_{1} p}+t^{1 / 4} e^{-\eta_{2} p} t^{1 / 2}+t^{1 / 4} e^{-\eta_{1} p}\right) \\
& \leq C t^{-1 / 4} e^{-\beta p} .
\end{aligned}
$$

Here we used that

$$
\begin{aligned}
\left\|\dot{u}_{1}^{h p}\right\|_{0,(-1,1)} & \leq\left\|\dot{u}_{1}-\dot{u}_{1}^{h p}\right\|_{0,(-1,1)}+\left\|\dot{u}_{1}\right\|_{0,(-1,1)} \\
& \leq C\left(t^{-1 / 4} e^{-\eta_{2} p}+t^{-1 / 2} t^{1 / 4}\right) \\
& \leq C t^{-1 / 4} \\
\left\|\dot{u}_{2}\right\|_{\infty} & =\left\|-\tilde{\lambda}_{0}(t) u_{2}\right\|_{\infty} \leq C t^{1 / 2}
\end{aligned}
$$

and that the derivative of $u_{1}$ with respect to $x$ is

$$
\dot{u}_{1}=-(1+i) \sqrt[4]{3\left(1-\nu^{2}\right)} t^{-1 / 2} u_{1} .
$$

In what follows we will treat separately each of the benchmark problems:

$\underline{\text { Case } 1}$ : both ends of the cylindrical shell are clamped (membrane dominated)

$\underline{\text { Case } 2}$ : free ends (bending dominated)

$\underline{\text { Case } 3}$ : simple sliding support (intermediate state).

\subsubsection{Case 1: clamped-clamped}

Theorem 3.6 If both ends of the cylindrical shell are clamped, then the following estimates for the relative error in the energy norm of the displacement field, smooth and boundary-layer components respectively hold:

$$
\begin{aligned}
\frac{\left\|\underline{U}-\underline{U}^{h p}\right\|}{\|\underline{U}\|} \leq C t^{-3 / 4} e^{-\eta p} \\
\frac{\left\|\underline{U}^{s}-\underline{U}^{s, h p}\right\|}{\left\|\mid \underline{U}^{s}\right\|} \leq C e^{-\eta p} \\
\frac{\left\|\underline{U}^{b}-\underline{U}^{b, h p}\right\|}{\left\|\underline{U}^{b}\right\|} \leq C t^{-1} e^{-\eta p}
\end{aligned}
$$


Remark 3.7 We notice that for this first benchmark problem we get the exponential rate of convergence (3.10) and the 'locking factor' $t^{-3 / 4}$, which is caused by the boundary-layer components. For the smooth component locking does not occur, according to (3.11). We see from (3.12), however, that the boundary-layer components cause membrane locking, even in membrane-dominated situations.

Proof. Let us first prove the estimates for the smooth and boundary-layer components, which together with (3.5) will imply immediately the estimate for the relative error of the displacement field $\underline{U}$.

Recall that we have already deduced in Lemma 3.2 the following bounds for the absolute errors in the 2-D energy norms in terms of the absolute errors in the $H^{1}$-norms of the 1-D reduced components:

$$
\begin{aligned}
\left\|\underline{U}^{s}-\underline{U}^{s, h p}\right\| & \leq C t^{1 / 2}\left[C_{\eta_{0}}\left\|\underline{u}^{s}-\underline{u}^{s, h p}\right\|_{1,(-1,1)} e^{-\eta_{0} p}+\left\|\underline{u}^{s}-\underline{u}^{s, h p}\right\|_{1,(-1,1)}\right. \\
& \left.+C_{\eta_{0}}\left\|\underline{u}^{s}\right\|_{1,(-1,1)} e^{-\eta_{0} p}\right] \\
\left\|\underline{U}^{b}-\underline{U}^{b, h p}\right\| & \leq C t^{1 / 2}\left[C_{\eta_{0}}\left\|\underline{u}^{b}-\underline{u}^{b, h p}\right\|_{1,(-1,1)} e^{-\eta_{0} p}+\left\|\underline{u}^{b}-\underline{u}^{b, h p}\right\|_{1,(-1,1)}\right. \\
& \left.+C_{\eta_{0}}\left\|\underline{u}^{b}\right\|_{1,(-1,1)} e^{-\eta_{0} p}\right]
\end{aligned}
$$

and notice that the equivalences of the 2-D and 1-D reduced energy norms imply also that

$$
\left\|\underline{U}^{s}\right\| \mid \sim\left\|\underline{u}^{s}\right\|_{e}, \quad\left\|\underline{U}^{b}\right\|\|\sim\| \underline{u}^{b} \|_{e} .
$$

Moreover, as proved in [11], the smooth component of the displacement field is in this case membrane-dominated, which implies that

$$
\left\|\underline{u}^{s}\right\|_{e} \geq C t^{1 / 2}\left\|\underline{u}^{s}\right\|_{1,(-1,1)} .
$$

Taking into account that

$$
\begin{gathered}
\left\|\underline{u}^{s}\right\|_{1,(-1,1)} \geq\left\|\underline{u}^{s}\right\|_{0,(-1,1)} \\
=\| \underline{u}^{0}+2 \operatorname{Re}\left(M(t)\left(e^{\lambda_{5}(t)(\cdot)} \mp e^{-\lambda_{5}(t)(\cdot)}\right) \|_{0,(-1,1)},\right. \\
\left\|\underline{u}^{0}\right\|_{0,(-1,1)} \geq C t^{-3},
\end{gathered}
$$

and

$$
C^{-1} t^{-5 / 2} \leq\|M(t)\| \leq C t^{-3},
$$


we find that

$$
\left\|\underline{u}^{s}\right\|_{1,(-1,1)} \geq C t^{-3}
$$

since

$$
\begin{aligned}
\left\|e^{\lambda_{5}(t)(\cdot)} \mp e^{-\lambda_{5}(t)(\cdot)}\right\|_{0,(-1,1)} & =\left[\int_{-1}^{1}\left(e^{2 \lambda_{5}(t) x}+e^{-2 \lambda_{5}(t) x} \mp 2\right) d x\right]^{1 / 2} \\
& =\left[\int_{-1}^{1} 2\left(e^{2 \lambda_{5}(t) x} \mp 1\right) d x\right]^{1 / 2} \\
& =\sqrt{2}\left[\frac{e^{2 \lambda_{5}(t)}-e^{-2 \lambda_{5}(t)}}{2 \lambda_{5}(t)} \mp 2\right]^{1 / 2} \\
& \geq C t^{1 / 2}
\end{aligned}
$$

which is a consequence of the asymptotic behaviour of the eigenvalue $\lambda_{5}(t)=O(\sqrt{t})$ and of the simple observation that $\left(e^{x}-e^{-x}\right) / x=2+x^{2} / 3+O\left(x^{4}\right)$. Therefore, Lemma 3.3 and the upper bound of the coefficient $\|M(t)\| \leq C t^{-3}$ imply now that

$$
\begin{aligned}
\frac{\left\|\underline{U}^{s}-\underline{U}^{s, h p}\right\|}{\left\|\underline{U}^{s}\right\|} & \leq C t^{1 / 2}\left[C_{\eta_{0}} \frac{\|M(t)\| e^{-\eta_{1} p}}{\left\|\underline{u}^{s}\right\|_{e}} e^{-\eta_{0} p}+\frac{\|M(t)\| e^{-\eta_{1} p}}{\left\|\underline{u}^{s}\right\|_{e}}+C_{\eta_{0}} \frac{\left\|\underline{u}^{s}\right\|_{1,(-1,1)}}{\left\|\underline{u}^{s}\right\|_{e}} e^{-\eta_{0} p}\right] \\
& \leq C_{\eta_{0}}\left(e^{-\eta_{1} p}+e^{-\eta_{0} p}\right) \\
& \leq C_{\eta_{0}} e^{-\min \left\{\eta_{1}, \eta_{0}\right\} p}
\end{aligned}
$$

which proves the second estimate of the theorem, with $\eta=\eta_{1}$, since we can choose $\eta_{0}>\eta_{1}$. Let us now prove the error estimate for the boundary-layer component, and recall for this the form of the reduced energy norm

$$
\begin{aligned}
\|\underline{u}\|_{e}^{2} & =\frac{t}{2} \int_{-1}^{1}\left[\nu\left(\beta_{11}+\beta_{22}\right)^{2}+(1-\nu) \sum_{i, j=1}^{2} \beta_{i j}^{2}\right] d x \\
& +\frac{t^{3}}{24} \int_{-1}^{1}\left[\nu\left(\kappa_{11}+\kappa_{22}\right)^{2}+(1-\nu) \sum_{i, j=1}^{2} \kappa_{i j}^{2}\right] d x+\frac{1-\nu}{4} t \int_{-1}^{1}\left(\rho_{1}^{2}+\rho_{2}^{2}\right) d x
\end{aligned}
$$

with $\beta_{i j}, \kappa_{i j}$ and $\rho_{i}$ defined in (2.4). Keeping from the energy norm only the term which involves the $H^{1}$-seminorm of the first component of the displacement field $\underline{u}^{b}=\left(u^{b}, v^{b}, w^{b}, \theta^{b}, \phi^{b}\right)$, we get the following lower bound:

$$
\left\|\underline{u^{b}}\right\|_{e} \geq C t^{1 / 2}\left|u^{b}\right|_{1,(-1,1)} \geq C t^{-1 / 4} .
$$

This can be deduced with appropriate estimates for the $L^{2}$-norms of the boundary-layers

$$
\left\|e^{-\lambda_{9}(t)(1-\cdot)}+e^{-\lambda_{9}(t)(1+\cdot)}\right\|_{0,(-1,1)} \geq C t^{1 / 2},
$$


respectively

$$
\left\|e^{-\lambda_{1}(t)(1-\cdot)}+e^{-\lambda_{1}(t)(1+\cdot)}\right\|_{0,(-1,1)} \geq C t^{1 / 4}
$$

and from the lower bounds of the first components of the coefficients $K_{1}(t)$, and $L_{1}(t)$ :

$$
\left|K_{1}(t)\right| \geq C t^{3}, \quad\left|L_{1}(t)\right| \geq C t^{-1 / 2} .
$$

Indeed, let us prove only (3.15); then (3.16) can be deduced analogously:

$$
\begin{aligned}
\left\|e^{-\lambda_{9}(t)(1-\cdot)}+e^{-\lambda_{9}(t)(1+\cdot)}\right\|_{0,(-1,1)} & =\left[\int_{-1}^{1}\left(e^{-2 \lambda_{9}(t)(1-x)}+e^{-2 \lambda_{9}(t)(1+x)}+2 e^{-2 \lambda_{9}(t)}\right) d x\right]^{1 / 2} \\
& =\left[2 \int_{-1}^{1}\left(e^{-2 \lambda_{9}(t)(1-x)}+e^{-2 \lambda_{9}(t)}\right) d x\right]^{1 / 2} \\
& =\left[2\left(\frac{1-e^{4 \lambda_{9}(t)}}{2 \lambda_{9}(t)}+2 e^{-2 \lambda_{9}(t)}\right)\right]^{1 / 2} \\
& \geq C t^{1 / 2} .
\end{aligned}
$$

Consequently, we get that

$$
\begin{aligned}
\left|u^{b}\right|_{1,(-1,1)}= & \left\|u^{b \prime}\right\|_{0,(-1,1)} \\
= & \| \lambda_{9}(t) K_{1}(t)\left(e^{-\lambda_{9}(t)(1-\cdot)}+e^{-\lambda_{9}(t)(1+\cdot)}\right) \\
& +2 \operatorname{Re}\left[\lambda_{1}(t) L_{1}(t)\left(\left(e^{-\lambda_{1}(t)(1-\cdot)}+e^{-\lambda_{1}(t)(1+\cdot)}\right)\right] \|_{0,(-1,1)}\right. \\
\geq & || \lambda_{9}(t)|| K_{1}(t) \mid\left\|e^{-\lambda_{9}(t)(1-\cdot)}+e^{-\lambda_{9}(t)(1+\cdot)}\right\|_{0,(-1,1)} \\
& -2\left|\lambda_{1}(t)\right|\left|L_{1}(t)\right|\left\|e^{-\lambda_{1}(t)(1-\cdot)}+e^{-\lambda_{1}(t)(1+\cdot)}\right\|_{0,(-1,1)} \mid .
\end{aligned}
$$

Estimating separately the boundary-layer components which correspond to the $t$ and $\sqrt{t}$ length scales,

$$
\left|\lambda_{9}(t)\right|\left|K_{1}(t)\right|\left\|e^{-\lambda_{9}(t)(1-\cdot)}+e^{-\lambda_{9}(t)(1+\cdot)}\right\|_{0,(-1,1)} \geq C t^{-1} \cdot t^{3} \cdot t^{1 / 2}=C t^{5 / 2}
$$

and

$$
\left|\lambda_{1}(t)\right|\left|L_{1}(t)\right|\left\|e^{-\lambda_{1}(t)(1-\cdot)}+e^{-\lambda_{1}(t)(1+\cdot)}\right\|_{0,(-1,1)} \geq C t^{-1 / 2} \cdot t^{-1 / 2} \cdot t^{1 / 4}=C t^{-3 / 4}
$$

we see that the $\sqrt{t}$ scale boundary-layer component dominates asymptotically. This leads us to the following estimates:

$$
\begin{aligned}
\left\|\underline{u}^{b}\right\|_{e} & \geq t^{1 / 2}\left|u^{b}\right|_{1,(-1,1)} \geq C t^{-1 / 4} \\
\left\|\underline{u}^{b}\right\|_{1,(-1,1)} & \leq C\left(\|K(t)\|\left\|e^{-\lambda_{9}(t)(1-\cdot)} \pm e^{-\lambda_{9}(t)(1+\cdot)}\right\|_{1,(-1,1)}\right.
\end{aligned}
$$




$$
\begin{aligned}
& \left.+\|L(t)\|\left\|e^{-\lambda_{1}(t)(1-\cdot)} \pm e^{-\lambda_{1}(t)(1+\cdot)}\right\|_{1,(-1,1)}\right) \\
\leq & C\left(t^{-1} t^{1 / 2}+t^{-3 / 2} t^{-1 / 2} t^{1 / 4}\right) \\
\leq & C t^{-7 / 4} .
\end{aligned}
$$

Here we made use of the fact that in this case the coefficients $K(t), L(t)$ have the following bounds : $\|K(t)\| \leq C,\|L(t)\| \leq C t^{-3 / 2}$, as shown in Table 1 .

Now it follows from the preceding estimates, Lemma 3.4 and Lemma 3.2 that the relative error of the boundary-layer component $\underline{U}^{b}$

$$
\begin{aligned}
\frac{\| \underline{\underline{U}^{b}-\underline{U}^{b, h p} \|}}{\left\|\underline{U}^{b}\right\|} \leq & C t^{1 / 2}\left[C_{\eta_{0}} \frac{\left\|\underline{u}^{b}-\underline{u}^{b, h p}\right\|_{1,(-1,1)}}{\left\|\underline{u}^{b}\right\|_{e}} e^{-\eta_{0} p}\right. \\
& \left.+\frac{\left\|\underline{u}^{b}-\underline{u}^{b, h p}\right\|_{1,(-1,1)}}{\left\|\underline{u}^{b}\right\|_{e}}+C_{\eta_{0}} \frac{\left\|\underline{u}^{b}\right\|_{1,(-1,1)}}{\left\|\underline{u}^{b}\right\|_{e}} e^{-\eta_{0} p}\right] \\
\leq & C t^{1 / 2}\left[C_{\eta_{0}} \frac{\|K(t)\| t^{-1 / 2}+\|L(t)\| t^{-1 / 4}}{t^{-1 / 4}} e^{-\beta p} e^{-\eta_{0} p}\right. \\
& \left.+\frac{\|K(t)\| t^{-1 / 2}+\|L(t)\| t^{-1 / 4}}{t^{-1 / 4}} e^{-\beta p}+C_{\eta_{0}} \frac{\left\|\underline{u}^{b}\right\|_{1,(-1,1)}}{\left\|\underline{u}^{b}\right\|_{e}} e^{-\eta_{0} p}\right] \\
\leq & C t^{1 / 2}\left[C_{\eta_{0}} \frac{t^{-1 / 2}+t^{-3 / 2} t^{-1 / 4}}{t^{-1 / 4}} e^{-\beta p} e^{-\eta_{0} p}+\frac{t^{-1 / 2}+t^{-3 / 2} t^{-1 / 4}}{t^{-1 / 4}} e^{-\beta p}\right] \\
& +C_{\eta_{0}} \frac{t^{-7 / 4}}{t^{-1 / 4}} e^{-\eta_{0} p} \\
\leq & C_{\eta_{0}} t^{-1} e^{-\min \left\{\beta, \eta_{0}\right\} p},
\end{aligned}
$$

which concludes the proof of Theorem 3.6, with $\eta=\beta$, since we can choose $\eta_{0}>\beta$.

\subsubsection{Case 2: free-free}

In this case we get similar estimates for the displacement field of the shell, smooth part and the boundary-layer component using the corresponding estimates for norms of the $x$-independent coefficients $K(t), L(t)$ and $M(t)$. We summarize them in the following

\section{Theorem 3.8}

$$
\begin{aligned}
\frac{\left\|\underline{U}-\underline{U}^{h p}\right\|}{\|\underline{U}\|} \leq C t^{-1} e^{-\eta p}, \\
\frac{\left\|\underline{U}^{s}-\underline{U}^{s, h p}\right\|}{\left\|\mid \underline{U}^{s}\right\|} \leq C t^{-1} e^{-\eta p}, \\
\frac{\left\|\underline{U}^{b}-\underline{U}^{b, h p}\right\|}{\left\|\underline{U}^{b}\right\|} \leq C t^{-1} e^{-\eta p} .
\end{aligned}
$$


Proof. As before, let us prove only the last two estimates, the relative error in the energy norm of $\underline{U}$ can then be deduced from these inequalities together with (3.5) and (3.6). With the absolute error estimates deduced in Lemma 3.2 we have that

$$
\begin{aligned}
\frac{\left\|\underline{U}^{s}-\underline{U}^{s, h p} \mid\right\|}{\left\|\underline{U}^{s}\right\|} \leq & C t^{1 / 2}\left[C_{\eta_{0}} \frac{\left\|\underline{u}^{s}-\underline{u}^{s, h p}\right\|_{1,(-1,1)}}{\left\|\underline{u}^{s}\right\|_{e}} e^{-\eta_{0} p}\right. \\
& \left.+\frac{\left\|\underline{u}^{s}-\underline{u}^{s, h p}\right\|_{1,(-1,1)}}{\left\|\underline{u}^{s}\right\|_{e}}+C_{\eta_{0}} \frac{\left\|\underline{u}^{s}\right\|_{1,(-1,1)}}{\left\|\underline{u}^{s}\right\|_{e}} e^{-\eta_{0} p}\right] .
\end{aligned}
$$

Then from Lemma 3.3 we know already that the absolute error in the $H^{1}$-norm of the smooth component $\underline{u}^{s}$ can be estimated by

$$
\left\|\underline{u}^{s}-\underline{u}^{s, h p}\right\|_{1,(-1,1)} \leq C\|M(t)\| e^{-\eta_{1} p} \leq C t^{-5 / 2} e^{-\eta_{1} p} .
$$

Furthermore, the smooth component $\underline{u}^{s}$ is in this case bending dominated [11], i.e. $\left\|\underline{u}^{s}\right\|_{e} \sim$ $t^{3 / 2}\left\|\underline{u}^{s}\right\|_{1,(-1,1)}$, which implies that $\left\|\underline{u}^{s}\right\|_{e} \geq C t^{-3 / 2}$, since

$$
\left\|\underline{u}^{s}\right\|_{1,(-1,1)} \geq\left|\left\|\underline{u}^{0}\right\|_{1,(-1,1)}-2\|M(t)\|\left\|e^{\lambda_{5}(t)(\cdot)} \mp e^{-\lambda_{5}(t)(\cdot)}\right\|_{1,(-1,1)}\right| \geq C t^{-3} .
$$

The last estimate is a consequence of the following bounds

$$
\begin{aligned}
C^{-1} t^{-2} & \leq\|M(t)\| \leq C t^{-5 / 2}, \\
C^{-1} t^{1 / 2} & \leq\left\|e^{\lambda_{5}(t)(\cdot)} \mp e^{-\lambda_{5}(t)(\cdot)}\right\|_{1,(-1,1)} \leq C .
\end{aligned}
$$

Therefore

$$
\begin{aligned}
\frac{\left\|\underline{\underline{U}^{s}}-\underline{U}^{s, h p}\right\| \mid}{\left\|\underline{\underline{U}^{s}}\right\|} \leq & C t^{1 / 2}\left[C_{\eta_{0}}\|M(t)\| \frac{1}{\left\|\underline{u}^{s}\right\|_{e}} e^{-\eta_{1} p} e^{-\eta_{0} p}\right. \\
& \left.+\|M(t)\| \frac{1}{\left\|\underline{u}^{s}\right\|_{e}} e^{-\eta_{1} p}+C_{\eta_{0}} \frac{\left\|\underline{u}^{s}\right\|_{1,(-1,1)}}{\left\|\underline{u}^{s}\right\|_{e}} e^{-\eta_{0} p}\right] \\
\leq & C_{\eta_{0}} t^{-1} e^{-\min \left\{\eta_{0}, \eta_{1}\right\} p},
\end{aligned}
$$

and this proves the second estimate of the theorem, with $\eta=\eta_{1}$, since we can choose $\eta_{0}>\eta_{1}$. For the last inequality we proceed again analogously as in the proof of Theorem 3.6 and take into account the corresponding bounds of the coefficients:

$$
\|K(t)\| \leq C t^{-3 / 2}, \quad\|L(t)\| \leq C t^{-5 / 2} .
$$

Next, as shown in Lemma 3.2

$$
\begin{aligned}
\frac{\left\|\underline{U}^{b}-\underline{U}^{b, h p}\right\| \mid}{\left\|\underline{U}^{b}\right\|} \leq & C t^{1 / 2}\left[C_{\eta_{0}} \frac{\left\|\underline{u}^{b}-\underline{u}^{b, h p}\right\|_{1,(-1,1)}}{\left\|\underline{u}^{b}\right\|_{e}} e^{-\eta_{0} p}\right. \\
& \left.+\frac{\left\|\underline{u}^{b}-\underline{u}^{b, h p}\right\|_{1,(-1,1)}}{\left\|\underline{u}^{b}\right\|_{e}}+C_{\eta_{0}} \frac{\left\|\underline{u}^{b}\right\|_{1,(-1,1)}}{\|\underline{u}\|_{e}} e^{-\eta_{0} p}\right],
\end{aligned}
$$


which can be further approximated with appropriate upper, respectively lower bounds for $\left\|\underline{u}^{b}-\underline{u}^{b, h p}\right\|_{1,(-1,1)}$ and $\left\|\underline{u}^{b}\right\|_{e}$.

Indeed, as it follows from Lemma 3.4 and (3.20)

$$
\begin{aligned}
\left\|\underline{u}^{b}-\underline{u}^{b, h p}\right\|_{1,(-1,1)} & \leq C\left(t^{-1 / 2} t^{-3 / 2}+t^{-5 / 2} t^{-1 / 4}\right) e^{-\beta p} \\
& \leq C t^{-11 / 4} e^{-\beta p}
\end{aligned}
$$

while

$$
\begin{aligned}
\left\|\underline{u}^{b}\right\|_{e} & \geq C t^{1 / 2}\left|u^{b}\right|_{1,(-1,1)} \\
& =C t^{1 / 2} \| \lambda_{9}(t) K_{1}(t)\left(e^{-\lambda_{9}(t)(1-\cdot)}+e^{-\lambda_{9}(t)(1+\cdot)}\right) \\
& +2 \operatorname{Re}\left[\lambda_{1}(t) L_{1}(t)\left(e^{-\lambda_{1}(t)(1-\cdot)}+e^{-\lambda_{1}(t)(1+\cdot)}\right)\right] \|_{0,(-1,1)} \\
& \geq|| \lambda_{9}(t)|| K_{1}(t) \mid\left\|e^{-\lambda_{9}(t)(1-\cdot)}+e^{-\lambda_{9}(t)(1+\cdot)}\right\|_{0,(-1,1)} \\
& -2\left|\lambda_{1}(t)\right|\left|L_{1}(t)\right|\left\|e^{-\lambda_{1}(t)(1-\cdot)}+e^{-\lambda_{1}(t)(1+\cdot)}\right\|_{0,(-1,1)} \mid .
\end{aligned}
$$

Estimating separately the boundary layer components which correspond to the $t$ and $\sqrt{t}$ length scales,

$$
\begin{aligned}
& \left|\lambda_{9}(t)\right|\left|K_{1}(t)\right|\left\|e^{-\lambda_{9}(t)(1-\cdot)}+e^{-\lambda_{9}(t)(1+\cdot)}\right\|_{0,(-1,1)} \geq C t^{-1} t^{3 / 2} t^{1 / 2}=C t \\
& \left|\lambda_{1}(t)\right|\left|L_{1}(t)\right|\left\|e^{-\lambda_{1}(t)(1-\cdot)}+e^{-\lambda_{1}(t)(1+\cdot)}\right\|_{0,(-1,1)} \geq C t^{-1 / 2} t^{-3 / 2} t^{1 / 4}=C t^{-7 / 4},
\end{aligned}
$$

since $|K(t)(1)| \geq C t^{3 / 2}$ and $|L(t)(1)| \geq C t^{-3 / 2}$. We see that the $\sqrt{t}$-scale boundary-layer component dominates asymptotically, which implies :

$$
\begin{aligned}
\left\|\underline{u}^{b}\right\|_{e} \geq & C t^{1 / 2}\left|u^{b}\right|_{1,(-1,1)} \geq C t^{-7 / 4} t^{1 / 2}=C t^{-5 / 4} \\
\left\|\underline{u}^{b}\right\|_{1,(-1,1)} \leq & C\left(\|K(t)\|\left\|e^{-\lambda_{9}(t)(1-\cdot)} \pm e^{-\lambda_{9}(t)(1+\cdot)}\right\|_{1,(-1,1)}\right. \\
& \left.+\|L(t)\|\left\|e^{-\lambda_{1}(t)(1-\cdot)} \pm e^{-\lambda_{1}(t)(1+\cdot)}\right\|_{1,(-1,1)}\right) \\
\leq & C\left(t^{-3 / 2} t^{-1} t^{1 / 2}+t^{-5 / 2} t^{-1 / 2} t^{1 / 4}\right) \\
\leq & C t^{-11 / 4} .
\end{aligned}
$$

This implies therefore that

$$
\begin{aligned}
\frac{\left\|\underline{U}^{b}-\underline{U}^{b, h p}\right\| \mid}{\left\|\underline{U}^{b} \mid\right\|} & \leq C t^{1 / 2}\left[C_{\eta_{0}} \frac{t^{-11 / 4}}{t^{-5 / 4}} e^{-\beta p} e^{-\eta_{0} p}+\frac{t^{-11 / 4}}{t^{-5 / 4}} e^{-\beta p}+C_{\eta_{0}} \frac{t^{-11 / 4}}{t^{-5 / 4}} e^{-\eta_{0} p}\right] \\
& \leq C_{\eta_{0}} t^{-1} e^{-\min \left\{\beta, \eta_{0}\right\} p},
\end{aligned}
$$

which concludes the proof of the last estimate with $\eta=\beta$, since we can take $\eta_{0}>\beta$. 


\subsubsection{Case 3: simple sliding support at the ends}

Theorem 3.9

$$
\begin{gathered}
\frac{\left\|\underline{U}-\underline{U}^{h p}\right\|}{\|\mid \underline{U}\|} \leq C t^{-1} e^{-\eta p}, \\
\frac{\left\|\underline{U}^{s}-\underline{U}^{s, h p}\right\|}{\left\|\underline{U}^{s}\right\|} \leq C t^{-1} e^{-\eta p}, \\
\frac{\left\|\underline{U}^{b}-\underline{U}^{b, h p}\right\|}{\left\|\underline{U}^{b}\right\|} \leq C t^{-1} e^{-\eta p},
\end{gathered}
$$

where the constants $C$ and $\eta>0$ do not depend on $t \in\left(0, t_{0}\right], \nu \in[0,1 / 2]$ and the polynomial degree $p$.

Proof. Let us split again the relative error of the displacement field $\underline{U}$ in the sum of the relative errors of its smooth, respectively boundary-layer parts:

$$
\frac{\left\|\underline{U}-\underline{U}^{h p} \mid\right\|}{\|\underline{U}\|} \leq A_{s} \frac{\left\|\underline{U}^{s}-\underline{U}^{s, h p}\right\| \mid}{\left\|\underline{U}^{s} \mid\right\|}+A_{b} \frac{\left\|\underline{U}^{b}-\underline{U}^{b, h p}\right\| \mid}{\left\|\underline{U}^{b}\right\|},
$$

where now the amplitudes

$$
A_{s} \sim t^{1 / 4} \quad \text { and } \quad A_{b} \sim 1 .
$$

In view of Lemma 3.2 and Lemma 3.3 the absolute error of the smooth component can be estimated as follows:

$$
\begin{aligned}
\left\|\underline{U}^{s}-\underline{U}^{s, h p} \mid\right\| \leq & C t^{1 / 2}\left[C_{\eta_{0}}\left\|\underline{u}^{s}-\underline{u}^{s, h p}\right\|_{1,(-1,1)} e^{-\eta_{0} p}+\left\|\underline{u}^{s}-\underline{u}^{s, h p}\right\|_{1,(-1,1)}\right. \\
& \left.+C_{\eta_{0}}\left\|\underline{u}^{s}\right\|_{1,(-1,1)} e^{-\eta_{0} p}\right] \\
\leq & C t^{1 / 2}\left[C_{\eta_{0}}\|M(t)\| e^{-\eta_{1} p} e^{-\eta_{0} p}+\|M(t)\| e^{-\eta_{1} p}+C_{\eta_{0}}\left\|\underline{u}^{s}\right\|_{1,1(-1,1)} e^{-\eta_{0} p}\right],
\end{aligned}
$$

so we get that

$$
\left\|\underline{U}^{s}-\underline{U}^{s, h p} \mid\right\| \leq C_{\eta_{0}} t^{1 / 2}\left(e^{-\eta_{1} p} t^{-4}+\left\|\underline{u}^{s}\right\|_{1,(-1,1)} e^{-\eta_{0} p}\right),
$$

since the coefficient $M(t)$ is in this case $\|M(t)\| \leq C t^{-4}$.

The smooth component of the displacement field is in this case membrane-dominated, which means that

$$
|| \underline{U}^{s}|| \sim\left\|\underline{u}^{s}\right\|_{e} \geq C t^{1 / 2}\left\|\underline{u}^{s}\right\|_{1,(-1,1)},
$$

where the last norm is

$$
\left\|\underline{u}^{s}\right\|_{1,(-1,1)}=\left\|\underline{u}_{0}+2 \operatorname{Re}\left(M(t)\left(e^{\lambda_{5}(t)(\cdot)} \mp e^{-\lambda_{5}(t)(\cdot)}\right)\right)\right\|_{1,(-1,1)} .
$$


Further, $\left\|\underline{u}^{0}\right\|_{0,(-1,1)} \sim t^{-3}$, and the lower bound for the coefficient $M(t), M(t) \geq C t^{-7 / 2}$ implies that:

$$
\begin{aligned}
\left\|2 \operatorname{Re}\left(M(t)\left(e^{\lambda_{5}(t)(\cdot)} \mp e^{-\lambda_{5}(t)(\cdot)}\right)\right)\right\|_{0,(-1,1)} & \geq C t^{-7 / 2}\left\|e^{\lambda_{5}(t)(\cdot)} \mp e^{-\lambda_{5}(t)(\cdot)}\right\|_{0,(-1,1)} \\
& \geq C t^{-3}
\end{aligned}
$$

since $\left\|e^{\lambda_{5}(t)(\cdot)} \mp e^{-\lambda_{5}(t)(\cdot)}\right\|_{0,(-1,1)} \geq C t^{1 / 2}$, as proved in (3.13). This means that

$$
\left\|\underline{u}^{s}\right\|_{0,(-1,1)} \geq C t^{-3}
$$

and because the $L^{2}$-norm dominates asymptotically, also

$$
\left\|\underline{u}^{s}\right\|_{1,(-1,1)} \geq C t^{-3} \text {. }
$$

Indeed,

$$
\begin{aligned}
\left|\underline{u}^{s}\right|_{1,(-1,1)} & =\| 2 \operatorname{Re}\left(M(t)\left(\lambda_{5}(t)\left(e^{\lambda_{5}(t)(\cdot)} \mp e^{-\lambda_{5}(t)(\cdot)}\right)\right) \|_{0,(-1,1)}\right. \\
& \geq C t^{-7 / 2} t^{1 / 2} t^{1 / 2}=C t^{-5 / 2} .
\end{aligned}
$$

Now follows the error estimate (3.24)

$$
\frac{\left\|\underline{U}^{s}-\underline{U}^{s, h p}\right\| \mid}{\left\|\underline{U}^{s} \mid\right\|} \leq C_{\eta_{0}} t^{-1} e^{-\min \left\{\eta_{1}, \eta_{0}\right\} p}
$$

with $\eta=\eta_{1}$, since we can assume without loss of generality that $\eta_{0}>\eta_{1}$.

For the estimate (3.25) we proceed analogously, using this time Lemma 3.4 and the corresponding upper and lower bounds for the coefficients $K(t)$ and $L(t)$, respectively.

As before,

$$
\begin{aligned}
\left\|\underline{U}^{b}-\underline{U}^{b, h p}\right\| \| & \leq C t^{1 / 2}\left\|\underline{U}^{b}-\underline{U}^{b, h p}\right\|_{1, \omega} \\
& \leq C t^{1 / 2}\left[C_{\eta_{0}}\left\|\underline{u}^{b}-\underline{u}^{b, h p}\right\|_{1,(-1,1)} e^{-\eta_{0} p}+\left\|\underline{u}^{b}-\underline{u}^{b, h p}\right\|_{1,(-1,1)}\right. \\
& \left.+C_{\eta_{0}}\left\|\underline{u}^{b}\right\|_{1,(-1,1)} e^{-\eta_{0} p}\right]
\end{aligned}
$$

and

$$
\begin{aligned}
\left\|\underline{u}^{b}-\underline{u}^{b, h p}\right\|_{1,(-1,1)} & \leq C\left(\|K(t)\| t^{-1 / 2}+\|L(t)\| t^{-1 / 4} \|\right) e^{-\beta p} \\
& \leq C\left(t^{-2} t^{-1 / 2}+t^{-5 / 2} t^{-1 / 4}\right) e^{-\beta p}=C t^{-11 / 4} e^{-\beta p}
\end{aligned}
$$




$$
\begin{aligned}
\left\|\underline{u}^{b}\right\|_{1,(-1,1)} & \leq C\left(\|K(t)\|\left\|e^{-\lambda_{9}(t)(1-\cdot)} \pm e^{-\lambda_{9}(t)(1+\cdot)}\right\|_{1,(-1,1)}\right. \\
& \left.+\|L(t)\|\left\|e^{-\lambda_{1}(t)(1-\cdot)} \pm e^{-\lambda_{1}(t)(1+\cdot)}\right\|_{1,(-1,1)}\right) \\
& \leq C\left(t^{-1 / 2} t^{-1} t^{1 / 2}+t^{-5 / 2} t^{-1 / 2} t^{1 / 4}\right) \\
& \leq C t^{-11 / 4}
\end{aligned}
$$

since $\|K(t)\| \leq C t^{-2}$ and $\|L(t)\| \leq C t^{-5 / 2}$ for this shell model. On the other hand, $\left\|\left|\underline{U}^{b}\right|\right\| \sim$ $\left\|\underline{u}^{b}\right\|_{e} \geq C t^{1 / 2}\left|u^{b}\right|_{1,(-1,1)}$, and the last seminorm can be further estimated from below as follows:

$$
\begin{aligned}
\left|u^{b}\right|_{1,(-1,1)} & =\left\|u^{b \prime}\right\|_{0,(-1,1)} \\
& =\| \lambda_{9}(t) K_{1}(t)\left(e^{-\lambda_{9}(t)(1-\cdot)}+e^{-\lambda_{9}(t)(1+\cdot)}\right) \\
& +2 \operatorname{Re}\left(\lambda_{1}(t) L_{1}(t)\left(e^{-\lambda_{1}(t)(1-\cdot)}+e^{-\lambda_{1}(t)(1+\cdot)}\right)\right) \|_{0,(-1,1)} \\
& \geq|| \lambda_{9}(t)\left|K_{1}(t)\right|\left\|e^{-\lambda_{9}(t)(1-\cdot)}+e^{-\lambda_{9}(t)(1+\cdot)}\right\|_{0,(-1,1)} \\
& -2\left|\lambda_{1}(t)\right| L_{1}(t)\left|\left\|e^{-\lambda_{1}(t)(1-\cdot)}+e^{-\lambda_{1}(t)(1+\cdot)}\right\|_{0,(-1,1)}\right| .
\end{aligned}
$$

Now, taking into account that $\left|K_{1}(t)\right| \geq C t$, and $\left|L_{1}(t)\right| \geq C t^{-3 / 2}$, we get that

$$
\begin{aligned}
& \left|\lambda_{9}(t)\right|\left|K_{1}(t)\right|\left\|e^{-\lambda_{9}(t)(1-\cdot)}+e^{-\lambda_{9}(t)(1+\cdot)}\right\|_{0,(-1,1)} \geq C t^{-1} t \cdot t^{1 / 2}=C t^{1 / 2}, \\
& \left|\lambda_{1}(t)\right|\left|L_{1}(t)\right|\left\|e^{-\lambda_{1}(t)(1-\cdot)}+e^{-\lambda_{1}(t)(1+\cdot)}\right\|_{0,(-1,1)} \geq C t^{-1 / 2} t^{-3 / 2} t^{1 / 4}=C t^{-7 / 4},
\end{aligned}
$$

and therefore

$$
\left|u^{b}\right|_{1,(-1,1)} \geq C t^{-7 / 4}
$$

Concluding,

$$
\begin{aligned}
\frac{\left\|\underline{\underline{U}^{b}}-\underline{U}^{b, h p}\right\|}{\left\|\underline{U^{b}}\right\|} & \leq C_{\eta_{0}} t^{1 / 2}\left(\frac{t^{-11 / 4}}{t^{1 / 2} t^{-7 / 4}} e^{-\beta p} e^{-\eta_{0} p}+\frac{t^{-11 / 4}}{t^{1 / 2} t^{-7 / 4}} e^{-\beta p}+\frac{t^{-11 / 4}}{t^{1 / 2} t^{-7 / 4}} e^{-\eta_{0} p}\right) \\
& \leq C_{\eta_{0}} t^{-1} e^{-\min \left\{\beta, \eta_{0}\right\} p}
\end{aligned}
$$

which is the last error estimate of the theorem, with $\eta=\beta<\eta_{0}$.

The last two estimates together with (3.5) imply now the first estimate.

\section{Numerical Results}

In this section we present numerical results for the solution of the cylindrical shell problem and confirm our theoretical results by the application of triangular and quadrilateral $h p$ fi- 
nite elements. In our computations we apply structured as well as unstructured meshes and confirm by these experiments the robustness of the $h p \mathrm{FE}$ technique for both triangular and quadrilateral elements.

We restrict our numerical examples to the bending dominated case 2, that is we assume free ends as boundary conditions, i.e. no kinematical constraints at the ends of the cylinder. This is the case which is strongly susceptible to membrane locking. The Poisson ratio $\nu$ is set to $\nu=1 / 3$ and the load that is applied to the cylinder is given by $f(x, \alpha)=\cos 2 \alpha$. This load is uniform in axial direction and equilibrated in angular direction, since there are rigid body modes present, which have to be eliminated. This is done by fixing the solution at three points by $U( \pm 1,0)=W( \pm 1,0)=\Theta( \pm 1,0)=0$ and $V(1, \pi / 4)=\Phi(1, \pi / 4)=0$. An exact solution for this cylindrical shell problem was obtained in Section 2.3.1. We point out that this system is ill conditioned (esp. for small $t$ ) but that it can be solved by using a program like MAPLE, which can compute with arbitrary high precision. The so obtained exact solution is the basis for our numerical convergence study.

We point out that in our numerical implementation [5] we do not exploit the symmetries of this model problem to reduce the computational domain. The whole cylinder is mapped into a planar rectangle, which is our computational domain. This rectangle is meshed as shown in Figure 1. The finite element mesh incorporates the $\sqrt{t}$ - and $t$-scale boundary layer elements for both triangular and quadrilateral elements. Figures 2 and 3 show the structured/unstructured triangular and quadrilateral $\mathrm{FE}$ meshes that we use for the shell thickness $t=0.01$. The numerical solution for the rotation $\Theta$ is also displayed in these Figures. The various boundary layers are clearly visible and well resolved by the chosen boundary layer elements. In the examples that correspond to Figures 2 and 3 the polynomial approximation order is $p=6$. The FE mesh consists of 48 quadrilaterals or of 96 triangles. The triangles are simply obtained by dividing the quadrilaterals along a diagonal. The polynomial approximation order can vary between 2 and 9 .

We emphasize that the cylinder has constant curvature coefficients and that we therefore can compute the stiffness matrix exactly by using a Gauss quadrature rule, which is exact for twice the polynomial degree of the shape functions. The integration error for the load vector is kept negligible by using a Gauss quadrature rule of order 19 .

In the following we present convergence rate plots for the energy for thicknesses $t=0.1$, $t=0.01$ and $t=0.001$. The thickness of the boundary layer elements is adjusted correspondingly to the shell thickness $t$, compare also Figure 1 . Figure 4 shows the convergence rates for the relative error in the energy for a structured triangular and a structured quadrilateral mesh. We clearly see that the $h p$ FEM delivers reliable numerical results and that the relative 

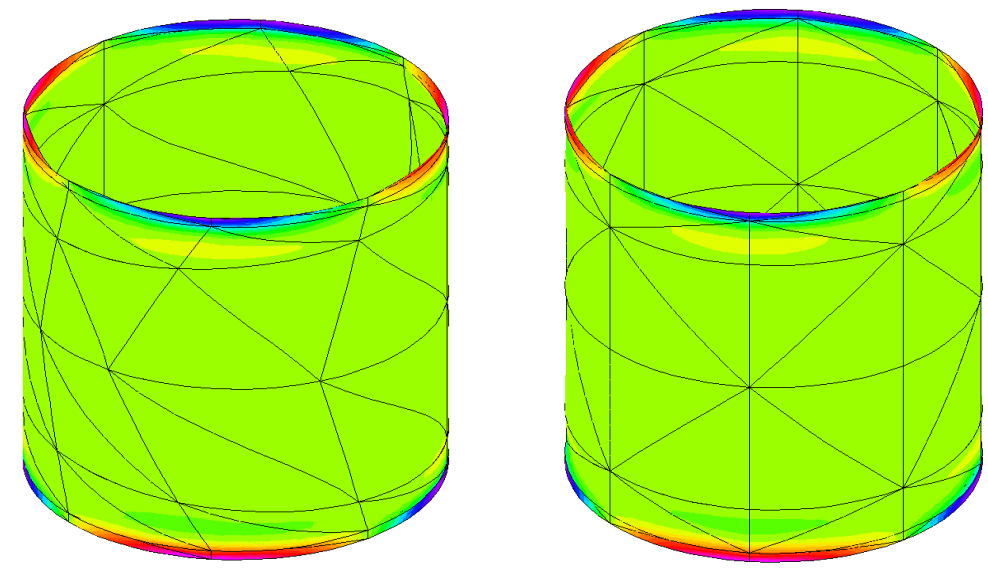

Figure 2: Rotation $\Theta$ on triangular meshes.
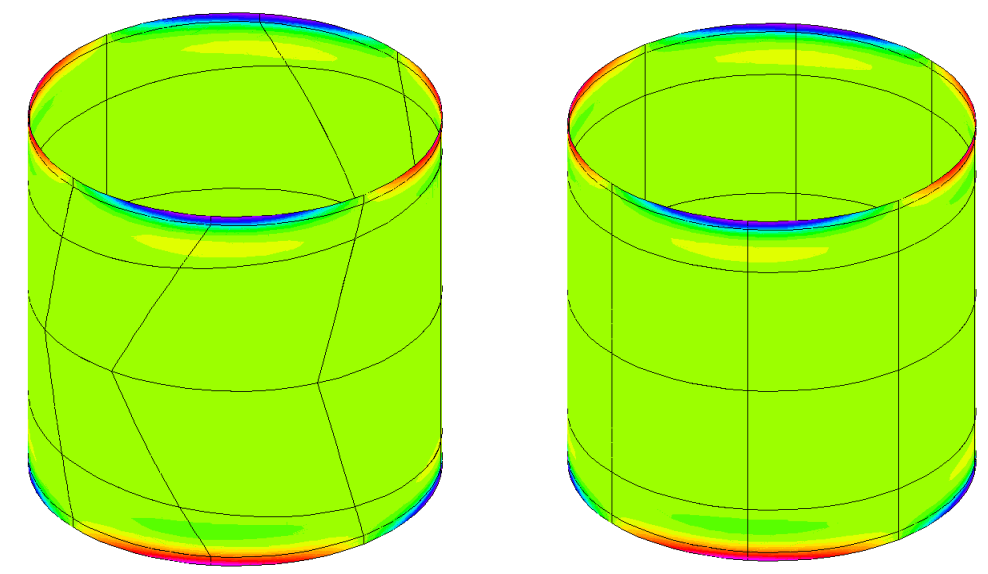

Figure 3: Rotation $\Theta$ on quadrilateral meshes. 

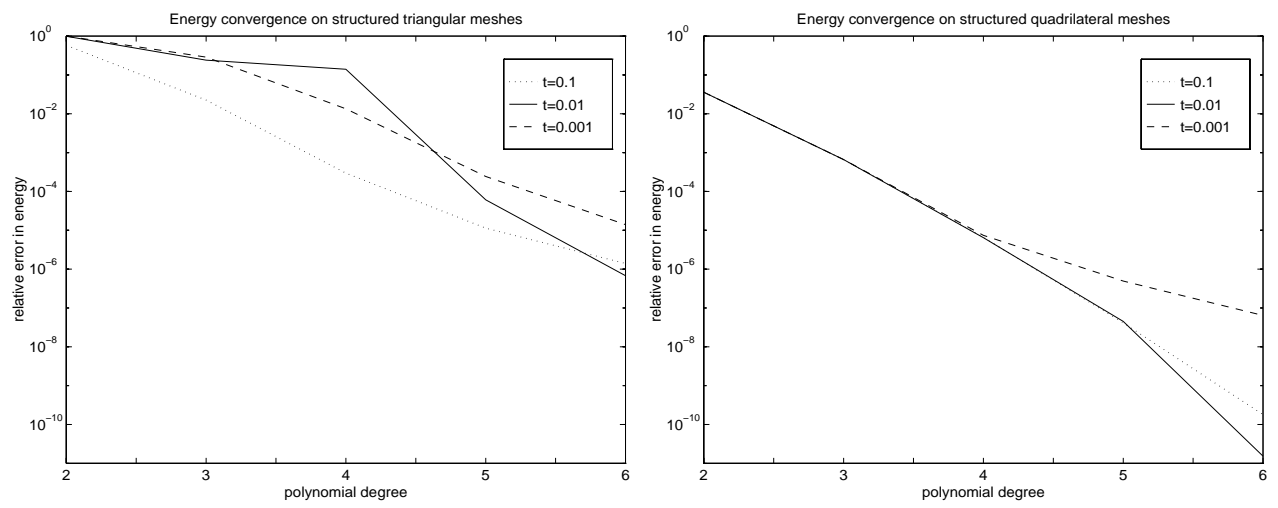

Figure 4: Energy convergence.

energy error is below $10^{-5}$ for $p=6$. In particular, we note that quadrilateral as well as triangular finite elements give similar results, although the quadrilaterals perform slightly better than the triangles. It is evident that the $h p$ approximation converges exponentially, which is consistent with our theoretical investigations. For very small thickness and high polynomial approximation order $(p>6)$, however, we run into conditioning problems. This observation is consistent with other numerical experiments that had been performed independently by using the commercial code STRESSCHECK.

The choice of the structured FE mesh is very specific for the cylindrical shell because the mesh is parallel and symmetric to the axis of the cylinder. For thickness $t=0.01$ we also use unstructured meshes, compare Figures 2 and 3. The energy convergence rates for both the structured and unstructured meshes are shown in Figure 5 for $t=0.01$. It is evident that the $h p$ approximation converges exponentially also on an unstructured mesh, which clearly indicates the robustness of the method with respect to mesh allignment. Obviously, the structured meshes perform better than the unstructured ones but there is no significant deterioration of the performance for the unstructured meshes. For the case of the structured quadrilateral mesh conditioning problems prevent the relative energy error from decaying further for $p>6$. For $p>6$ the error slightly increases due to the numerical roundoff errors that result from the ill conditioning of the problem. The conditioning problem also influences the numerical results on the other meshes at $t=10^{-3}$.

Now we focus on the quality of our numerical solution in the context of membrane locking. In detail, we want to study how the quality of the numerical approximation deteriorates with decreasing shell thickness $t$. Therefore, we define the locking factor for the energy, which is 


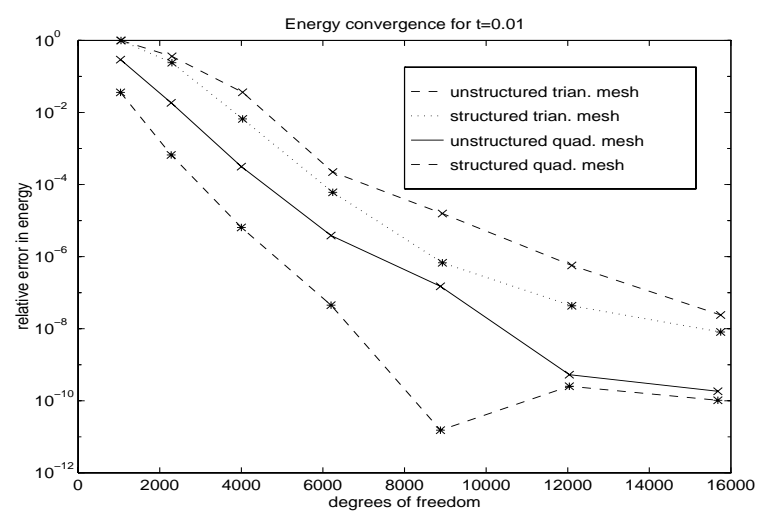

Figure 5: Energy convergence on structured and unstructured meshes for $t=0.01$.

the ratio of the relative error in energy for thickness $t$ and for thickness $t=1$, i.e.

$$
\kappa=\frac{\left|\mathcal{F}_{R}\left(\underline{\tilde{U}}^{h p} ; t\right)-\mathcal{F}_{R}(\underline{U} ; t)\right|}{\left|\mathcal{F}_{R}(\underline{U} ; t)\right|} / \frac{\left|\mathcal{F}_{R}\left(\underline{\tilde{U}}^{h p} ; 1\right)-\mathcal{F}_{R}(\underline{U} ; 1)\right|}{\left|\mathcal{F}_{R}(\underline{U} ; 1)\right|} .
$$

This ratio can be computed for various approximation orders $p$ and is tabulated in Table 2 for structured triangular meshes and in Table 3 for structured quadrilateral meshes. The locking factors are large for fine meshes and $t<<1$ which is a result of the fact that the $h p$ FEM with high polynomial approximation order solves the shell problem for $t=1$ extremely well. Basically all significant digits of the energy $\mathcal{F}_{R}\left(\underline{\tilde{U}}^{h p} ; 1\right)$ are correct, which gives a very small number in the denominator of (4.1) and results in a large locking factor. This also indicates that the convergence of the FEM for $t<<1$ is much slower than for $t=1$. The convergence rates for small $t$ do have a defect when compared to the convergence rates for $t=1$. This is expressed by the locking factors but we emphasize that the exponential convergence of the method still dominates, although significant locking factors are present. These locking factors are much larger for other quantities of engineering interest, such as stresses and strains, as we have seen from our numerical experiments.

\section{Conclusions}

In this paper, we have analyzed theoretically and numerically the $h p$-FEM for a Naghdi-type model of a cylindrical shell with various boundary conditions. It was found that with proper mesh design the boundary layers (resp. edge effects) could be resolved by inserting a single layer of anisotropic $p$-elements along the edge for each length-scale in the solution. The relative error in the energy was found to converge in all cases, from membrane dominated solutions to fully 


\begin{tabular}{|r|r|r|r|}
\hline $\mathrm{p}$ & $\mathrm{t}=0.1$ & $\mathrm{t}=0.01$ & $\mathrm{t}=0.001$ \\
\hline 2 & 5.69 & 9.52 & 9.47 \\
\hline 3 & 11.64 & 120.23 & 143.24 \\
\hline 4 & 16.90 & 351.75 & 710.61 \\
\hline 5 & 121.57 & 500.70 & $1.9910^{3}$ \\
\hline 6 & $3.5310^{3}$ & $1.3110^{3}$ & $2.3810^{4}$ \\
\hline 7 & $1.2610^{5}$ & $2.4110^{4}$ & $7.9110^{6}$ \\
\hline 8 & $1.5010^{5}$ & $4.4910^{4}$ & $9.8810^{7}$ \\
\hline
\end{tabular}

Table 2: Energy Locking Table. Triangular Structured Mesh.

\begin{tabular}{|r|r|r|r|}
\hline $\mathrm{p}$ & $\mathrm{t}=0.1$ & $\mathrm{t}=0.01$ & $\mathrm{t}=0.001$ \\
\hline 2 & 1.22 & 1.22 & 1.22 \\
\hline 3 & 1.22 & 1.23 & 1.23 \\
\hline 4 & 1.22 & 1.20 & 1.38 \\
\hline 5 & 1.22 & 1.33 & 14.52 \\
\hline 6 & 1.22 & 0.11 & 434.41 \\
\hline 7 & 2.67 & 534.80 & $1.2710^{6}$ \\
\hline 8 & 84.27 & $6.3810^{3}$ & $8.4310^{8}$ \\
\hline
\end{tabular}

Table 3: Energy Locking Table. Quadrilateral Structured Mesh.

bending dominated solutions, exponentially in terms of the degrees of freedom, however, with a locking factor $O\left(t^{\beta}\right)$ with $-1 \leq \beta \leq 0$. It was found that the exponential convergence rate compensates for possible adverse impact of the locking factor so that various case-dependent devices (as, e.g., reduced integration) aiming at a reduction of membrane locking are not required.

We have seen that the shell boundary layers can be resolved in the $h p$-approach by inserting for each length scale one layer of long elements of width $O\left(p_{\max } t\right)$ near the edge where $t$ is the length scale of the layer to be resolved. For the Naghdi-type shells under consideration here, 2 length scales, $O(t)$ and $O(\sqrt{t})$, were present. For other geometric situations, also $O(\sqrt[3]{t})$ and $O(\sqrt[4]{t})$ are present $[12,15]$. These layers can be resolved using the same strategy.

In this work, only the case of a cylindrical shell was analyzed. The $h p$-FE discretization presented, however, is completely general and applies to any shell-geometry, e.g. on hyperbolic or elliptic shells, provided exact parameter representations of the shell mid-surface are available. Our implementation also has a complete $h p$ capability, i.e. the polynomial approximation order cannot only vary from element to element but can also vary within each element. So called hanging nodes can also be handled by this particular implementation for the shell problem, 
which is based on a flexible $h p$ code described in [5]. A similar convergence analysis could be performed for general shells with smooth midsurfaces once sufficiently refined asymptotics of the exact solutions of the shell models are available. Present rapid progress [4] raises the hope that this may be so in the near future.

Some issues that will be addressed in forthcoming work are: in practice, additional $h p$ surface approximation must be performed whose impact on the solution accuracy is not known; this holds likewise for the order of numerical integration for the different parts of the stiffness matrix. Finally, there is of course the issue of the modeling error between the exact solutions of the shell model and of three-dimensional elasticity. While there are now asymptotic a-priori estimates of the error between three-dimensional and two-dimensional linearly elastic models (see [4], and the references there), much work needs to be done on the computable a-posteriori estimation of the shell modeling error. Any such estimate requires, however, an accurate numerical solution of the shell model itself (see [13] for an analysis of the plate case). Here, the $h p$-FEM seems to be an attractive alternative to other, more classical approaches, or to approaches tailored specifically to bending dominated shells.

\section{A Appendix}

Theorem A.1 The roots of the characteristic polynomial

$$
\begin{aligned}
P(\lambda ; t, \nu) & =3 t^{4} \lambda^{10}+\left(-6 t^{4} \nu-2 t^{6} \nu-2 t^{6}-36 t^{2}-78 t^{4}\right) \lambda^{8} \\
& +\left(24 \nu^{2} t^{4}-36 t^{2} \nu^{2}+72 t^{2} \nu+16 t^{6} \nu+72 t^{4} \nu+684 t^{2}+16 t^{6}+504 t^{4}\right) \lambda^{6} \\
& +\left(-2880 t^{2}-24 t^{6}-288 t^{2} \nu-120 t^{4} \nu-1416 t^{4}-8 t^{6} \nu+16 \nu^{2} t^{6}-432+432 \nu^{2}\right) \lambda^{4} \\
& +\left(5184 t^{2}+2160 t^{4}\right) \lambda^{2}-1728 t^{4}-5184 t^{2}
\end{aligned}
$$

can be expanded in Puiseux series with respect to $t \in\left(0, t_{0}\right]$, for a sufficiently small $t_{0}>0$, such that the principal parts are analytic as functions of $t,\left(\right.$ for $\left.\lambda_{i}(t), i=9,10\right)$, or as a function of $\sqrt{t},\left(\right.$ for $\lambda_{i}(t), i=1, \ldots, 8$ respectively).

Proof. Let us start by rewriting the characteristic polynomial in the following form:

$$
P(\lambda ; t, \nu)=3 t^{4} \lambda^{10}+t^{2} a(t) \lambda^{8}+t^{2} b(t) \lambda^{6}+c(t) \lambda^{4}+t^{2} d(t) \lambda^{2}+t^{2} e(t),
$$

where $a(t), b(t), c(t), d(t)$ and $e(t)$ are polynomials with respect to $t$, such that

$$
a(0) \neq 0, \quad b(0) \neq 0, \quad c(0) \neq 0, \quad d(0) \neq 0, \quad e(0) \neq 0 .
$$


We substitute then

$$
\lambda^{2}=x \text { and } x=y / t^{4},
$$

so that the characteristic equation becomes:

$$
3 y^{5}+a(t) t^{2} y^{4}+b(t) t^{6} y^{3}+c(t) t^{8} y^{2}+d(t) t^{14} y+e(t) t^{18}=0 .
$$

We already expect that two of the roots, $\lambda_{9}(t)$ and $\lambda_{10}(t)$, are Laurent series with respect to $t$, and their singular parts are of the form $\alpha \cdot 1 / t$, for some constant $\alpha$, so we further make the substitution $x=z / t^{2}$.

This implies $y=t^{2} z$, and the characteristic equation becomes

$$
\begin{aligned}
3\left(t^{2} z\right)^{5}+a(t) t^{2}\left(t^{2} z\right)^{4}+b(t) t^{6}\left(t^{2} z\right)^{3}+c(t) t^{8}\left(t^{2} z\right)^{2}+d(t) t^{14}\left(t^{2} z\right)+e(t) t^{18} & =0 \\
\Leftrightarrow \quad 3 t^{10} z^{5}+a(t) t^{10} z^{4}+b(t) t^{12} z^{3}+c(t) t^{12} z^{2}+d(t) t^{16} z+e(t) t^{18} & =0 .
\end{aligned}
$$

Dividing by $t^{10}$ we get

$$
Q(z ; t):=3 z^{5}+a(t) z^{4}+b(t) t^{2} z^{3}+c(t) t^{2} z^{2}+d(t) t^{6} z+e(t) t^{8}=0 .
$$

Now, obviously $Q(z ; t)$ is an analytic function in both variables, and

$$
Q(z ; 0)=3 z^{5}+a(0) z^{4}=z^{4}(3 z+a(0)) .
$$

If we take $z_{0}=-a(0) / 3 \neq 0$, then

$$
Q\left(z_{0} ; 0\right)=0, \quad \frac{\partial Q}{\partial z}\left(z_{0} ; 0\right) \neq 0
$$

which allow us to apply the Implicit Function Theorem (analytic version), to get that $\exists z(\cdot)=$ $z(t) \in \mathcal{A}((-\varepsilon, \varepsilon))$, for some $\varepsilon>0$ sufficiently small, such that

$$
Q(z(t) ; t)=0, \forall t \in(-\varepsilon, \varepsilon), z(0)=z_{0}=12>0 .
$$

This implies $z(t)>0, \forall t \in(-\varepsilon, \varepsilon)$, for sufficiently small $\varepsilon>0$, and an immediate consequence of this is that also $\sqrt{z(t)} \in \mathcal{A}((-\varepsilon, \varepsilon))$. Then

$$
\lambda_{9}(t)=\frac{\sqrt{z(t)}}{t}, \quad \lambda_{10}(t)=-\frac{\sqrt{z(t)}}{t},
$$

and this proves our claim for these eigenvalues.

For the other eigenvalues we proceed further analogously. We already expect that the singular part of the first four, $\lambda_{i}(t), i=1, . ., 4$, is of the form $\beta \cdot 1 / \sqrt{t}$, for some constant $\beta$, so we 
substitute again in the characteristic equation $x=z / t$. This means that $y=t^{4} x=t^{3} z$. In this way the equation becomes:

$$
3\left(t^{3} z\right)^{5}+t^{2} a(t)\left(t^{3} z\right)^{4}+b(t) t^{6}\left(t^{3} z\right)^{3}+c(t) t^{8}\left(t^{3} z\right)^{2}+d(t) t^{14}\left(t^{3} z\right)+e(t) t^{18}=0,
$$

so if we divide both sides by $t^{14}$ we get

$$
Q_{1}(z ; t):=3 t z^{5}+a(t) z^{4}+b(t) t z^{3}+c(t) z^{2}+d(t) t^{3} z+e(t) t^{4}=0
$$

We notice again that $Q_{1}(z ; t)$ is an analytic function in both variables, and

$$
Q_{1}(z ; 0)=a(0) z^{4}+c(0) z^{2}=c(0) z^{2}\left(1+\frac{a(0)}{c(0)} z^{2}\right)
$$

has $z_{01}=i \sqrt{c(0) / a(0)}$ and $-z_{01}$ as simple roots $\left(c(0)=-432\left(1-\nu^{2}\right)<0\right.$ for $\left.\nu \in[0,1 / 2]\right)$ :

$$
\begin{aligned}
Q_{1}\left(z_{01} ; 0\right) & =0, & Q_{1}\left(-z_{01} ; 0\right) & =0 \\
\frac{\partial Q_{1}}{\partial z}\left(z_{01} ; 0\right) & \neq 0, & \frac{\partial Q_{1}}{\partial z}\left(-z_{01} ; 0\right) & \neq 0 .
\end{aligned}
$$

Next, the Implicit Function Theorem implies that $\exists z_{1}=z_{1}(t) \in \mathcal{A}((-\varepsilon, \varepsilon)), \varepsilon$ eventually smaller, such that

$$
\begin{array}{rlrlrl}
Q_{1}\left(z_{1}(t) ; t\right) & =0, & Q_{1}\left(\bar{z}_{1}(t) ; t\right) & =0, & & \forall t \in(-\varepsilon, \varepsilon), \\
z_{1}(0) & =z_{01}, & \operatorname{Im}\left(z_{1}(t)\right)>0, & & \forall t \in(-\varepsilon, \varepsilon) .
\end{array}
$$

Then the first four eigenvalues are:

$$
\begin{aligned}
& \lambda_{1}(t)=\frac{1}{\sqrt{2 t}}(1+i) \sqrt{\operatorname{Im}\left(z_{1}(t)\right)-i \operatorname{Re}\left(z_{1}(t)\right)}, \\
& \lambda_{2}(t)=\bar{\lambda}_{1}(t) \\
& \lambda_{3}(t)=-\lambda_{1}(t), \\
& \lambda_{4}(t)=-\bar{\lambda}_{1}(t),
\end{aligned}
$$

with the choice of the principal branch of the square root function with complex arguments, i.e. $\sqrt{z}=\sqrt{|z|} \exp (i \arg z / 2), \arg z \in(-\pi, \pi)$.

It remains to analyze now the eigenvalues $\lambda_{i}(t), i=5, . .8$, which we expect to be of the form $\sqrt{t} \cdot f(t)$, with $f(t)$ an analytic function in a small neighbourhood of 0 . Then, take $x=t z$, which implies $y=t^{4} x=t^{5} z$, and substitute this in the characteristic equation to get

$$
3\left(t^{5} z\right)^{5}+a(t) t^{2}\left(t^{5} z\right)^{4}+b(t) t^{6}\left(t^{5} z\right)^{3}+c(t) t^{8}\left(t^{5} z\right)^{2}+d(t) t^{14}\left(t^{5} z\right)+e(t) t^{18}=0 .
$$


Dividing both sides of the preceding equation by $t^{18}$ it follows:

$$
Q_{2}(z ; t):=3 t^{7} z^{5}+a(t) t^{4} z^{4}+b(t) t^{3} z^{3}+c(t) z^{2}+d(t) t z+e(t)=0 .
$$

It is easy to see now that $Q_{2}(z ; t)$ is an analytic function in $z$ and $t$, and the equation $Q_{2}(z ; 0)=$ $c(0) z^{2}+e(0)$ has two simple, complex conjugated roots $z_{02}=i \sqrt{e(0) / c(0)},-z_{02},(e(0)=$ $-5184<0)$ :

$$
\begin{aligned}
& Q_{2}\left(z_{02} ; 0\right)=Q_{2}\left(-z_{02} ; 0\right)=0, \\
& \frac{\partial Q_{2}}{\partial z}\left(z_{02} ; 0\right), \frac{\partial Q_{2}}{\partial z}\left(z_{02} ; 0\right) \neq 0 .
\end{aligned}
$$

Then, eventually taking $\varepsilon>0$ smaller, we find that $\exists z_{2}(t) \in \mathcal{A}((-\varepsilon, \varepsilon))$, such that

$$
\begin{aligned}
Q_{2}\left(z_{2}(t) ; t\right)=Q_{2}\left(\bar{z}_{2}(t) ; t\right) & =0, \forall t \in(-\varepsilon, \varepsilon), \\
z_{2}(0) & =z_{02},
\end{aligned}
$$

and because of the continuity of the implicit solution we can also assume that

$$
\operatorname{Im}\left(z_{2}(t)\right)>0, \forall t \in(-\varepsilon, \varepsilon) .
$$

Then the remaining eigenvalues are

$$
\begin{aligned}
& \lambda_{5}(t)=\sqrt{\frac{t}{2}}(1+i) \sqrt{\operatorname{Im}\left(z_{2}(t)\right)-i \operatorname{Re}\left(z_{2}(t)\right)}, \\
& \lambda_{6}(t)=\bar{\lambda}_{5}(t), \\
& \lambda_{7}(t)=-\lambda_{5}(t), \\
& \lambda_{8}(t)=-\bar{\lambda}_{5}(t),
\end{aligned}
$$

with the same choice of the square root in the complex context $\sqrt{z}=\sqrt{|z|} \exp (i \arg z / 2)$, $\arg z \in(-\pi, \pi)$, so the theorem is completely proved.

\section{References}

[1] I. Babuška, M. Suri, "The $p$ and $h-p$ versions of the finite element method, basic principles and properties", SIAM review 36 (1994) 578-632.

[2] K.J. Bathe, F. Brezzi and M. Fortin, "Mixed-interpolated elements for Reissner-Mindlin plates", Int. J. Numer. Methods Engrg. 28 (1989) 1787-1801. 
[3] D. H. Brown Associates, Inc., "CAE: Ready for the next Leap Forward; A Survey of Trends", Mechanical CAD/CAM Research Program, 222 Grace Church Street, Port Chester, NY 10573, USA.

[4] P. G. Ciarlet and V. Lods, "Asymptotic Analysis of linearly elastic shells I: Justification of membrane shell equations", Arch. Rat. Mech. Anal. 136 (1996) 119-161.

[5] A. Bajer, L. Demkowicz, K. Gerdes, C. Schwab, T. Walsh " $h p$-FEM for general variational problems in 2D", in preparation.

[6] J. Sanchez-Hubert, E. Sanchez-Palencia, "Coques élastiques minces. Propriétés asymptotiques", Recherche en mathematique appliquées, Masson, Paris, 1997.

[7] W. T. Koiter, "On the foundations of the linear theory of thin elastic shells", Proc. Kon. Ned. Akad. Wetensch., B73(1970) 169-195.

[8] L.S.D. Morley, "Analysis of developable shells with special reference to the finite element method and circular cylinders", Phil. Trans. R. Soc. Lond. A 281 (1976) 113-170.

[9] J. Piila, J. Pitkäranta, "Energy estimates relating different linear elastic models of a thin cylindrical shell; I. The membrane-dominated case", SIAM J. Math. Anal. Vol. 24, No. 1, January 1993, 1-22.

[10] J. Pitkäranta, "The problem of membrane locking in finite element analysis of cylindrical shells", Numer. Math. 61 (1992) 523-542.

[11] J. Pitkäranta, Y. Leino, O. Ovaskainen, J. Piila, "Shell deformation states and the finite element method: A benchmark study of cylindrical shells", Comput. Methods Appl. Mech. Engrg. 128 (1995), 81-121.

[12] J. Pitkäranta, C. Schwab, "Resolution of shell boundary layers by $h p$-FEM", in preparation.

[13] C. Schwab, "A-posteriori modeling error estimation for hierarchic plate models", Num. Math. 74(1996) 221-259.

[14] C. Schwab, M. Suri, C.A. Xenophontos, "Boundary Layer Approximation by Spectral/hp Methods", ICOSAHOM'95: Proceedings of the Third International Conference on Spectral and High Order Methods, University of Houston, Journal of Mathematics, Houston, $1996,501-508$. 
[15] C. Schwab, M. Suri, C. Xenophontos, "The $h p$ finite element method for problems in mechanics with boundary layers", SAM Report 96-20, in press in Computer Methods in Applied Mechanics and Engineering.

[16] C. Schwab, M. Suri, "The $p$ and $h p$ versions of the Finite Element Method for problems with boundary layers", Mathematics of Computation, vol 65, no 216, oct 1996, 1403-1429.

[17] B. Szabo and I. Babuška, Finite Element Analysis, Wiley 1991. 